\title{
Lumped Elastodynamic Model for MEMS: Formulation and Validation
}

\author{
Philippe Cardou, Member, IEEE, Member, ASME, Damiano Pasini, Member, IEEE, Member, ASME, \\ and Jorge Angeles, Fellow, IEEE, Fellow, ASME
}

\begin{abstract}
Proposed in this paper is a symbolic linearly elastodynamic model for the analysis and synthesis of microelectromechanical system. In particular, the strain energy in the compliant links is computed from the results of a previous work, by representing beam deflections with small-displacement screws. This allows for a systematic coherent approach based on screw theory. Two case studies are proposed to illustrate the application of the model. In the first example, the elastodynamic model of a simple accelerometer is derived and compared to one available in the literature. In the second example, a complex accelerometer is modeled, fabricated, and tested. Comparison between the simulated results and data from the literature or obtained experimentally shows the accuracy of the proposed model.

[2007-0287]
\end{abstract}

Index Terms-Accelerometer, analysis, compliant mechanism, cross-axial motions, dynamics, mechanical systems, stiffness matrix, synthesis.

\section{INTRODUCTION}

$\mathbf{T}$ HE DEVELOPMENT of micromachining techniques has led to the realization of more sophisticated microelectromechanical-system (MEMS) devices (see [1] and [2]). In most instances, the underlying mechanisms are modeled numerically [3], either through finite-element methods [4] or others, such as finite differences [5], relaxation methods [6], or the rigid finite-element method [7]. These methods have been incorporated to numerical-synthesis methods, among which we may cite the ground-structure method [8], the load-pathsynthesis approach [9], and the homogenization method [10]. If these methods apply to a broad range of problems, each solution gives information regarding the single problem that corresponds to the chosen objective function, loads, constraints, mesh, optimization domain, etc. Hence, these numerical methods tend to bury the physics of MEMS under the data. In contrast, when possible, a symbolic approach yields mathematical relations that deepen the insight of the designer. This is thought to be important, particularly at the early stage of the design

Manuscript received November 27, 2007; revised April 2, 2008. First published July 18, 2008; last published August 1, 2008 (projected). This work was supported by Fonds Québécois de Reserche sur la Nature et les Technologies. The work of P. Cardou was supported in part by NSERC under a Canada Graduate Scholarship and in part by FQRNT under Research Grant PR-112531. Subject Editor N. Aluru.

P. Cardou is with the Robotics Laboratory, Department of Mechanical Engineering, Laval University, Quebec, PQ GIK 7P4, Canada (e-mail: pcardou@ gmc.ulaval.ca).

D. Pasini and J. Angeles are with the Department of Mechanical Engineering, McGill University, Montreal, PQ H3A 1Y1, Canada (e-mail: damiano.pasini@ mcgill.ca; angeles@cim.mcgill.ca).

Color versions of one or more of the figures in this paper are available online at http://ieeexplore.ieee.org.

Digital Object Identifier 10.1109/JMEMS.2008.927178 process. Accordingly, the method proposed in this paper may be regarded as a complement to numerical-synthesis methods. The classical approach for the synthesis of compliant micromechanisms is to simplify the representation of the physical problem by making a number of assumptions, which come at the expense of model accuracy. As the proposed method targets the early synthesis of MEMS, simplicity of application to a wide range of design variants is thought to be more important than model accuracy, leaving the final dimensional optimization to more accurate numerical methods. Hence, several of the classical assumptions of elastodynamic modeling will be adopted in the formulation as follows.

Such assumptions are at the root of other symbolic models for compliant MEMS. One instance of this is the replacement of the compliant links with two or more rigid links articulated with one or more kinematic pairs-generally prismatic or revolute - constrained by translational or torsional springs. This is the idea behind the pseudorigid-body model [11]-[13]. The main merit of this method is to be accurate over large displacements while remaining relatively simple, thus allowing for a symbolic approach. However, this method is difficult to implement when it comes to arbitrary displacements in space. Indeed, the pseudorigid-body model requires that the springdriven kinematic pairs be inserted at specific positions on the compliant links of the mechanism. If that link is to deflect about several directions and axes - in translation and rotation-then several kinematic pairs need to be added in series at different positions, which results in a complex kinematics model.

Another common assumption is that of small displacements of the micromechanism rigid links from their reference poses, which allows the linearization of their dynamics [14]. This assumption is valid in most MEMS applications, since the small displacements involved tend to affect important quantities such as air friction, heat dissipation, and electrostatic forces but generally have little effect on the inertia and stiffness properties of the mechanism. Hence, the small-displacement assumption will be adopted here.

From the foregoing observations, it would be interesting to have a systematic method for deriving symbolically the complete linearized elastodynamics of complex lumpedcompliance micromechanisms undergoing small displacements. To this end, we resort to screw theory. We build upon the work reported in [15], which describes the elastic properties of beams by representing their deformations with small-displacement screws. From these results, we are able to compute the strain energy in a compliant link from the smalldisplacement screw representing the relative displacement of 


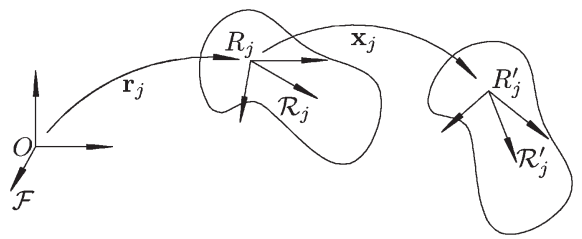

Fig. 1. $j$ th rigid link in its equilibrium pose and in a displaced pose.

the two rigid links it connects. The resulting model pertains to the linear theory of elastodynamics [16] and should be relevant to the analysis and synthesis of complex micromechanisms (e.g., [2] and [17]).

Two examples are included to illustrate the application of the proposed model. The first example treats the simple case of the ADXL150, an accelerometer produced by Analog Devices; comparison of the results obtained with those reported in [18] is included. The second example addresses the elastodynamics modeling of a complex micromechanism composed of 4 rigid and 18 compliant links. The model obtained is compared with experimental results.

\section{Dynamic Model of Lumped-Compliance Mechanisms Undergoing SMALl Displacements}

Since this is a lumped-parameter model, each component of the flexible mechanism falls into one of two categories. The first category gathers the $m$ compliant links, which are assumed to have no inertia and a given nonnull compliance in all directions. The second category contains the $n$ rigid links, which are attributed a given inertia and no compliance.

Moreover, the compliant links are modeled as EulerBernoulli beams, and all the rigid-link displacements are considered to be small. From this last assumption, the mass and stiffness properties of the links are assumed to be constant, i.e., independent of the mechanism posture.

\section{A. System Posture}

Let us first define the fixed frame $\mathcal{F}$ and frame $\mathcal{R}_{j}^{\prime}$ attached to the $j$ th rigid link, as shown in Fig. 1. Moreover, we define frame $\mathcal{R}_{j}$ as coinciding with frame $\mathcal{R}_{j}^{\prime}$ whenever the $j$ th rigid body lies in its equilibrium pose, designated its reference pose. The origins of frames $\mathcal{F}, \mathcal{R}_{j}$, and $\mathcal{R}_{j}^{\prime}$ are labeled as $O, R_{j}$, and $R_{j}^{\prime}, j=1, \ldots, n$, respectively, where $R_{j}$ is chosen to lie at the mass center of its corresponding rigid link.

The displacement taking $\mathcal{F}$ into $\mathcal{R}_{j}$ is described by the pose array $\mathbf{r}_{j} \equiv\left[\begin{array}{ll}\boldsymbol{\theta}_{j}^{\mathrm{T}} & \boldsymbol{\rho}_{j}^{\mathrm{T}}\end{array}\right]^{\mathrm{T}}$, where $\boldsymbol{\theta}_{j} \in \mathbb{R}^{3}$ is defined as the product of the natural invariants [19] $\theta_{j}$ and $\mathbf{d}_{j}$ of the associated rotation, and $\boldsymbol{\rho}_{j} \equiv \overrightarrow{O R_{j}} \in \mathbb{R}^{3}$. The natural invariants of a rotation are the unit-vector $\mathbf{d}_{j}$ pointing in the direction of its associated axis, and its angle of rotation $\theta_{j}$. In order to avoid ambiguities, we use the right-hand rule in order to determine the direction of the rotation around the screw axis, and we constrain $\boldsymbol{\theta}_{j}$ within a ball of radius $\pi$, i.e., $0 \mathrm{rad} \leq\left\|\boldsymbol{\theta}_{j}\right\|_{2} \leq \pi \mathrm{rad}$. Notice that this leaves an ambiguity at $\left\|\boldsymbol{\theta}_{j}\right\|_{2}=\pi \mathrm{rad}$, since in that case, $\boldsymbol{\theta}_{j}$ and $-\boldsymbol{\theta}_{j}$ yield the same rotation. However, since we are using these parameters to describe postures of the mechanism that are close to its static-equilibrium posture, this ambiguity may be resolved a priori by the good judgment of the designer.

Similarly, we define the pose of the $j$ th rigid body with respect to its equilibrium pose as

$$
x_{j} \equiv\left[\begin{array}{ll}
\nu_{j}^{\mathrm{T}} & \xi_{j}^{\mathrm{T}}
\end{array}\right]^{\mathrm{T}}
$$

where $\boldsymbol{\nu}_{j} \in \mathbb{R}^{3}$ is the array of products of the natural invariants for the rotation taking $\mathcal{R}_{j}$ into $\mathcal{R}_{j}^{\prime}$ and following the same convention as that used for $\boldsymbol{\theta}_{j}$; moreover, $\boldsymbol{\xi}_{j} \equiv \overrightarrow{R_{j} R_{j}} \in \mathbb{R}^{3}$. Since the posture of the mechanism is fully described by the poses of all the rigid links, we define the $6 n$-dimensional posture array

$$
x \equiv\left[\begin{array}{llll}
x_{1}^{\mathrm{T}} & x_{2}^{\mathrm{T}} & \cdots & x_{n}^{\mathrm{T}}
\end{array}\right]^{\mathrm{T}} .
$$

\section{B. System Kinetic Energy}

For starters, we need an expression for the angular velocity $\boldsymbol{\omega}_{j}$ of the $j$ th link, which is known to be linear in the derivatives of the linear invariants [19]. We start by computing rather $\boldsymbol{\Omega}_{j}$, the angular-velocity matrix of the $j$ th link, defined as the product $\mathbf{Q}_{j} \mathbf{Q}_{j}^{\mathrm{T}}$, with $\mathbf{Q}_{j}$ denoting the rotation matrix that carries $\mathcal{F}$ into an orientation identical to that of $\mathcal{R}_{j}$, making abstraction of the translation of the origin. In fact, $\boldsymbol{\Omega}_{j}$ is $\operatorname{CPM}\left(\boldsymbol{\omega}_{j}\right)$, with $\operatorname{CPM}(\cdot)$ denoting the cross-product matrix ${ }^{1}$ of the 3-D Cartesian vector $(\cdot)$. Once $\boldsymbol{\Omega}_{j}$ is found, $\boldsymbol{\omega}_{j}$ is readily derived as its axial vector ${ }^{2} \operatorname{vect}\left(\boldsymbol{\Omega}_{j}\right)$.

The expression for $\mathbf{Q}_{j}$ in terms of the linear invariants is recalled for quick reference [19]

$$
\mathbf{Q}_{j}=\mathbf{d}_{j} \mathbf{d}_{j}^{\mathrm{T}}+\cos \theta_{j}\left(1-\mathbf{d}_{j} \mathbf{d}_{j}^{\mathrm{T}}\right)+\sin \theta_{j} \operatorname{CPM}\left(\mathbf{d}_{j}\right)
$$

in which one is the $3 \times 3$ identity matrix. Under the "small displacement" assumption, $\theta_{j}$ is small enough so that $\cos \theta_{j} \approx 1$ and $\sin \theta_{j} \approx \theta_{j}$; the foregoing expression thus reducing to

$$
\mathbf{Q}_{j} \approx 1+\theta_{j} \operatorname{CPM}\left(\mathbf{d}_{j}\right) \equiv 1+\operatorname{CPM}\left(\theta_{j} \mathbf{d}_{j}\right) \equiv 1+\mathbf{N}_{j}
$$

where $\mathbf{N}_{j} \equiv \operatorname{CPM}\left(\boldsymbol{v}_{j}\right)$.

Hence, the product $\mathbf{Q}_{j} \mathbf{Q}_{j}^{\mathrm{T}}$, which yields $\boldsymbol{\Omega}_{j}$, can be approximated as

$$
\boldsymbol{\Omega}_{j} \approx \mathbf{N}_{j}\left(1+\mathbf{N}_{j}^{\mathrm{T}}\right)=\mathbf{N}_{j}+\mathbf{N}_{j} \mathbf{N}_{j}^{\mathrm{T}} .
$$

The first term of the foregoing expression is bilinear in the natural invariants and their time derivative; the second is quadratic in the former, linear in the latter. Under our "smalldisplacement" assumption, then, we drop the second term and end up with an approximation for $\boldsymbol{\Omega}_{j}$ involving only the first term of the foregoing expression, whence the approximation of $\boldsymbol{\omega}_{j}$ follows:

$$
\omega_{j} \approx \nu_{j}, \quad j=1, \ldots, n .
$$

\footnotetext{
${ }^{1} \mathrm{CPM}(\mathbf{a})$ is defined as $\partial(\mathbf{a} \times \mathbf{x}) / \partial \mathbf{x}$, for any $\mathbf{a}, \mathbf{x} \in \mathbb{R}^{3}$.

${ }^{2}$ The axial vector vect $(\mathbf{A})$ of a $3 \times 3$ matrix $\mathbf{A}$ is defined, for every 3-D vector $\mathbf{x}$, as the $3-\mathrm{D}$ vector $\mathbf{a}$ for which $(1 / 2)\left(\mathbf{A}+\mathbf{A}^{\mathrm{T}}\right) \mathbf{x} \equiv \operatorname{CPM}(\mathbf{a}) \mathbf{x} \equiv$ $\mathbf{a} \times \mathbf{x}$.
} 


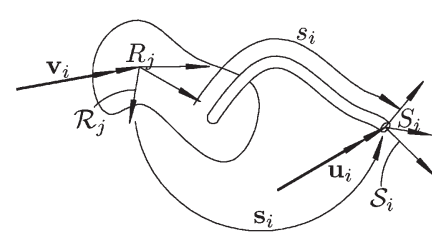

(a)

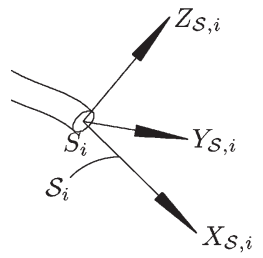

(b)
Fig. 2. $i$ th compliant link attached to the $j$ th rigid link. (a) Layout. (b) Detail of the definition of $\mathcal{S}_{i}\left(s_{i}\right)$.

Let us store the mass properties of the $j$ th rigid link into its associated inertia dyad [19]

$$
\mathbf{M}_{j} \equiv\left[\begin{array}{cc}
\mathbf{I}_{j} & \mathbf{O} \\
\mathbf{O} & m_{j} \mathbf{1}
\end{array}\right]
$$

where $m_{j}$ is the mass of the $j$ th rigid link, $\mathbf{I}_{j}$ is its inertia matrix about point $R_{j}$, its mass center, and $\mathbf{O}$ is the $3 \times 3$ zero-matrix. As a result, the kinetic energy $T$ of the system is computed as

$$
T=\frac{1}{2} \sum_{j=1}^{n} \dot{\mathbf{x}}_{j}^{\mathrm{T}} \mathbf{M}_{j} \dot{\mathbf{x}}_{j}=\frac{1}{2} \dot{\mathbf{x}}^{\mathrm{T}} \mathbf{M} \dot{\mathbf{x}}
$$

where

$$
\mathbf{M} \equiv\left[\begin{array}{cccc}
\mathbf{M}_{1} & \mathbf{O}_{6 \times 6} & \cdots & \mathbf{O}_{6 \times 6} \\
\mathbf{O}_{6 \times 6} & \mathbf{M}_{2} & \cdots & \mathbf{O}_{6 \times 6} \\
\vdots & \vdots & \ddots & \vdots \\
\mathbf{O}_{6 \times 6} & \mathbf{O}_{6 \times 6} & \cdots & \mathbf{M}_{n}
\end{array}\right]
$$

will be referred to as the mass matrix of the mechanism.

\section{System Potential Energy}

Consider the $i$ th compliant link that is clamped, at one end, to the $j$ th rigid link and, at the other end, to the $k$ th rigid link, with $j<k$. From the free-body diagram of the $i$ th compliant link shown in Fig. 2(a), we see that the wrench $\mathbf{v}_{i} \in \mathbb{R}^{6}$ applied at the mass center $R_{j}$ by the $j$ th rigid link onto the $i$ th compliant link has to be balanced out by wrench $\mathbf{u}_{i}\left(s_{i}\right) \in \mathbb{R}^{6}$ applied at point $S_{i}\left(s_{i}\right)$, where $s_{i}$ is a curvilinear coordinate along the beam neutral axis. The wrenches are defined so that their reciprocal product with the small-displacement screws, defined in (1), is dimensionally meaningful. Therefore, the first three components of the wrench represent a moment, whereas the last three represent a force; the latter applied at the corresponding mass center, where the wrench is defined. Let us attach frame $\mathcal{S}_{i}\left(s_{i}\right)$ with axes $X_{\mathcal{S}, i}, Y_{\mathcal{S}, i}$, and $Z_{\mathcal{S}, i}$ to the beam cross section at $s_{i}$, as shown in Fig. 2(b). Frame $\mathcal{S}_{i}\left(s_{i}\right)$ is defined so as to have its $X_{\mathcal{S}, i}$-axis tangent to the beam neutral axis and pointing in the positive direction of $s_{i}$, and its $Y_{\mathcal{S}, i^{-}}$and $Z_{\mathcal{S}, i}$-axes along the principal directions of the cross section. Let $\tau_{i}\left(s_{i}\right)$ be the array of products of the natural invariants of the rotation taking frame $\mathcal{R}_{j}$ into frame $\mathcal{S}_{i}\left(s_{i}\right)$, following the same convention as that used for $\boldsymbol{\theta}_{j}$, and $\boldsymbol{\sigma}_{i}\left(s_{i}\right) \in \mathbb{R}^{3}$ be the vector moving point $R_{j}$ into $S_{i}\left(s_{i}\right)$. We regroup these two arrays in the cross-sectional pose array

$$
\mathbf{s}_{i}\left(s_{i}\right) \equiv\left[\begin{array}{ll}
\boldsymbol{\tau}_{i}\left(s_{i}\right)^{\mathrm{T}} & \boldsymbol{\sigma}_{i}\left(s_{i}\right)^{\mathrm{T}}
\end{array}\right]^{\mathrm{T}} \in \mathbb{R}^{6} .
$$

Furthermore, let us define $\boldsymbol{\Sigma}_{i} \equiv \operatorname{CPM}\left(\boldsymbol{\sigma}_{i}\right)$ and $\mathbf{T}_{i} \equiv$ $\operatorname{CPM}\left(\boldsymbol{\tau}_{i}\right)$.

The strain energy in a beam element of length $d s_{i}$ starting at coordinate $s_{i}$ and ending at coordinate $s_{i}+d s_{i}$ is computed as [15]

$$
d U_{i}\left(s_{i}\right)=\frac{1}{2}\left[\mathbf{u}_{i}\left(s_{i}\right)\right]_{\mathcal{S}, i}^{\mathrm{T}} \mathbf{H}_{i}\left(s_{i}\right)\left[\mathbf{u}_{i}\left(s_{i}\right)\right]_{\mathcal{S}, i} d s_{i}
$$

where $[\cdot]_{\mathcal{S}, i}$ indicates that the quantity $(\cdot)$ is expressed in frame $\mathcal{S}_{i}$ and with respect to its origin $S_{i}$. Matrix $\mathbf{H}_{i}\left(s_{i}\right) \in \mathbb{R}^{6 \times 6}$, in turn, contains the properties of the cross section. This matrix is defined according to the strain-energy formulas for beams [20]

$$
\mathbf{H}_{i}\left(s_{i}\right) \equiv \operatorname{diag}\left(\frac{1}{G_{i} J_{i}}, \frac{1}{E_{i} I_{Y, i}}, \frac{1}{E_{i} I_{Z, i}}, \frac{1}{E_{i} A_{i}}, \frac{\alpha_{Y, i}}{G_{i} A_{i}}, \frac{\alpha_{Z, i}}{G_{i} A_{i}}\right)
$$

where $E$ and $G$ are the Young and the shear moduli, respectively; $I_{Y, i}, I_{Z, i}$, and $J_{i}$ are the $Y_{\mathcal{S}, i}$-axis moment of inertia, the $Z_{\mathcal{S}, i}$-axis moment of inertia, and the torsional modulus of the beam cross section, respectively ${ }^{3} ; A_{i}$ is the area of the cross section; and $\alpha_{Y, i}$ and $\alpha_{Z, i}$ are the shearing-effect coefficients for the $Y_{\mathcal{S}, i^{-}}$and $Z_{\mathcal{S}, i}$-directions, respectively. Notice that all these parameters are functions of the curvilinear coordinate $s_{i}$.

In the sequel, we shall need the adjoint representation of the Euclidean group [21], which maps linearly the associated Lie algebra onto itself. In the case of the cross-sectional pose-array screw $\mathbf{s}_{i}\left(s_{i}\right)$, we obtain

$$
\mathbf{S}_{i} \equiv\left[\begin{array}{cc}
e^{\mathbf{T}_{i}\left(s_{i}\right)} & \mathbf{O}_{3 \times 3} \\
\boldsymbol{\Sigma}_{i}\left(s_{i}\right) e^{\mathbf{T}_{i}\left(s_{i}\right)} & e^{\mathbf{T}_{i}\left(s_{i}\right)}
\end{array}\right]
$$

which leads to the following expression of wrench $\mathbf{v}_{i}$ in frame $\mathcal{S}_{i}$, namely,

$$
\left[\mathbf{u}_{i}\left(s_{i}\right)\right]_{\mathcal{S}, i}=-\left[\mathbf{v}_{i}\right]_{\mathcal{S}, i}=-\mathbf{S}_{i}^{\mathrm{T}}\left[\mathbf{v}_{i}\right]_{\mathcal{R}, j}
$$

where the first equality was obtained from the equilibrium in the free-body diagram of Fig. 2(a). Upon substituting (12) into (9) and integrating over the length of the $i$ th compliant link, we obtain the strain energy as

$$
U_{i}=\frac{1}{2}\left[\mathbf{v}_{i}\right]_{\mathcal{R}, j}^{\mathrm{T}} \mathbf{B}_{i}\left[\mathbf{v}_{i}\right]_{\mathcal{R}, j}
$$

where

$$
\mathbf{B}_{i} \equiv \int_{0}^{l_{i}} \mathbf{S}_{i}\left(s_{i}\right) \mathbf{H}_{i}\left(s_{i}\right) \mathbf{S}_{i}\left(s_{i}\right)^{\mathrm{T}} d s_{i}
$$

and $l_{i}$ is the length of the $i$ th compliant link. It will prove useful to express all wrenches $\mathbf{v}_{i}$ in the same reference frame $\mathcal{F}$.

\footnotetext{
${ }^{3} I_{Y, i}, I_{Z, i}$, and $J$ are defined with respect to the centroid of the cross section.
} 
To do this, we will need the adjoint representation of the Euclidean group that corresponds to the rigid-body motion taking frame $\mathcal{F}$ into frame $\mathcal{R}_{j}$, namely,

$$
\mathbf{R}_{j} \equiv\left[\begin{array}{cc}
e^{\Theta_{i}} & \mathbf{O} \\
\mathbf{\Upsilon}_{i} e^{\Theta_{i}} & e^{\Theta_{i}}
\end{array}\right]
$$

where $\boldsymbol{\Theta}_{i} \equiv \operatorname{CPM}\left(\boldsymbol{\theta}_{i}\right)$ and $\boldsymbol{\Upsilon}_{i} \equiv \operatorname{CPM}\left(\boldsymbol{\rho}_{i}\right)$. Hence,

$$
\left[\mathbf{v}_{i}\right]_{\mathcal{R}, j}=\mathbf{R}_{j}^{\mathrm{T}}\left[\mathbf{v}_{i}\right]_{\mathcal{F}}
$$

and the total strain energy-or potential energy, for that matter-of the system becomes

$$
U=\sum_{i=1}^{m} U_{i}=\sum_{i=1}^{m} \frac{1}{2}\left[\mathbf{v}_{i}\right]_{\mathcal{F}}^{\mathrm{T}} \mathbf{R}_{j} \mathbf{B}_{i} \mathbf{R}_{j}^{\mathrm{T}}\left[\mathbf{v}_{i}\right]_{\mathcal{F}}
$$

For the sake of conciseness, let us rewrite this expression as

$$
U=\frac{1}{2}[\mathbf{v}]_{\mathcal{F}}^{\mathrm{T}} \mathbf{B}[\mathbf{v}]_{\mathcal{F}}
$$

where $\mathbf{B}$ is a block-diagonal matrix, namely,

$$
\mathbf{B} \equiv \operatorname{diag}\left\{\mathbf{R}_{j_{1}} \mathbf{B}_{1} \mathbf{R}_{j_{1}}^{\mathrm{T}}, \mathbf{R}_{j_{2}} \mathbf{B}_{2} \mathbf{R}_{j_{2}}^{\mathrm{T}}, \ldots, \mathbf{R}_{j_{m}} \mathbf{B}_{m} \mathbf{R}_{j_{m}}^{\mathrm{T}}\right\}_{i=1}^{m}
$$

with $j_{i}$ taking the value of the smallest index among those of the two rigid links that are connected to the $i$ th compliant link, and

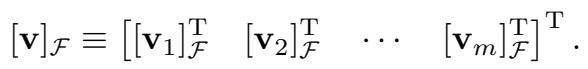

Upon writing the static equilibrium of the wrenches acting on the $j$ th rigid link, we obtain

$$
\left[\mathbf{w}_{j}\right]_{\mathcal{R}_{j}}-\sum_{i \in \mathcal{C}_{j}^{+}}\left[\mathbf{v}_{i}\right]_{\mathcal{R}_{j}}+\sum_{i \in \mathcal{C}_{j}^{-}}\left[\mathbf{v}_{i}\right]_{\mathcal{R}_{j}}=\mathbf{0}_{6}
$$

where $\mathbf{0}_{6}$ is the 6-D zero-vector and $\mathcal{C}_{j}^{+}$is the set of the indexes of the compliant links that are connected to the $j$ th rigid link and to another rigid link that has an index greater than $j$, while $\mathcal{C}_{j}^{-}$is the set of the indexes of the compliant links that are connected to the $j$ th rigid link and to another rigid link that has an index smaller than $j$. We substitute (15) into (18) and solve for $\left[\mathbf{w}_{j}\right]_{\mathcal{R}_{j}}$, which leads to

$$
\left[\mathbf{w}_{j}\right]_{\mathcal{R}_{j}}=\mathbf{R}_{j}^{\mathrm{T}}\left(\sum_{i \in \mathcal{C}_{j}^{+}}\left[\mathbf{v}_{i}\right]_{\mathcal{F}}-\sum_{i \in \mathcal{C}_{j}^{-}}\left[\mathbf{v}_{i}\right]_{\mathcal{F}}\right) .
$$

In order to simplify the notation, we define the arrays $\mathbf{v}$ and $\mathbf{w}$ of compliant- and rigid-link wrenches, respectively, i.e.,

$$
[\mathbf{v}]_{\mathcal{F}} \equiv\left[\begin{array}{c}
{\left[\mathbf{v}_{1}\right]_{\mathcal{F}}} \\
{\left[\mathbf{v}_{2}\right]_{\mathcal{F}}} \\
\vdots \\
{\left[\mathbf{v}_{m}\right]_{\mathcal{F}}}
\end{array}\right] \quad[\mathbf{w}]_{\mathcal{R}} \equiv\left[\begin{array}{c}
{\left[\mathbf{w}_{1}\right]_{\mathcal{R}, 1}} \\
{\left[\mathbf{w}_{2}\right]_{\mathcal{R}, 2}} \\
\vdots \\
{\left[\mathbf{v}_{n}\right]_{\mathcal{R}, n}}
\end{array}\right]
$$

Accordingly, we may rewrite (19) in the more convenient form

$$
[\mathbf{w}]_{\mathcal{R}}=\mathbf{R}^{\mathrm{T}} \mathbf{A}[\mathbf{v}]_{\mathcal{F}}
$$

where $\mathbf{R} \equiv \operatorname{diag}\left\{\mathbf{R}_{1}, \mathbf{R}_{2}, \ldots, \mathbf{R}_{n}\right\} \in \mathbb{R}^{6 n \times 6 n}$, while

$$
\mathbf{A} \equiv\left[\begin{array}{cccc}
\mathbf{A}_{11} & \mathbf{A}_{12} & \cdots & \mathbf{A}_{1 m} \\
\mathbf{A}_{21} & \mathbf{A}_{22} & \cdots & \mathbf{A}_{2 m} \\
\vdots & \vdots & \ddots & \vdots \\
\mathbf{A}_{n 1} & \mathbf{A}_{n 2} & \cdots & \mathbf{A}_{n m}
\end{array}\right] \in \mathbb{R}^{6 n \times 6 m}
$$

Moreover

$$
\mathbf{A}_{j i}= \begin{cases}\mathbf{O}_{6 \times 6} & \begin{array}{l}
\text { if compliant-link } i \text { is not connected } \\
\text { to rigid-link } j
\end{array} \\
1_{6 \times 6} & \begin{array}{l}
\text { if compliant-link } i \text { is connected to } \\
\text { rigid-links } j \text { and } k, \text { with } j<k
\end{array} \\
-1_{6 \times 6} & \begin{array}{l}
\text { if compliant-link } i \text { is connected to } \\
\text { rigid-links } j \text { and } k, \text { with } j>k .
\end{array}\end{cases}
$$

This allows the introduction of the potential energy of the external wrenches as a function of the internal wrenches, namely,

$$
\Pi=-[\mathbf{w}]_{\mathcal{R}}^{\mathrm{T}}[\mathbf{x}]_{\mathcal{R}}=-[\mathbf{v}]_{\mathcal{F}}^{\mathrm{T}} \mathbf{A}^{\mathrm{T}} \mathbf{R}[\mathbf{x}]_{\mathcal{R}}
$$

For a linearly elastic system, the potential energy $V$ and the complementary potential energy $\bar{V}$ take the same value, which is the sum of the strain energy and the potential energy, namely,

$$
\bar{V}=V=U+\Pi=(1 / 2)[\mathbf{v}]_{\mathcal{F}}^{\mathrm{T}} \mathbf{B}[\mathbf{v}]_{\mathcal{F}}-[\mathbf{v}]_{\mathcal{F}}^{\mathrm{T}} \mathbf{A}^{\mathrm{T}} \mathbf{R}[\mathbf{v}]_{\mathcal{R}} .
$$

The internal wrenches $\mathbf{v}$ are computed from the minimization of the potential energy $V$ for given displacements $\mathbf{x}$ of the rigid links. This follows from the second theorem of Castigliano. The partial derivative of $\bar{V}$ with respect to the internal wrenches yields

$$
\frac{\partial \bar{V}}{\partial[\mathbf{v}]_{\mathcal{F}}}=\mathbf{B}[\mathbf{v}]_{\mathcal{F}}-\mathbf{A}^{\mathrm{T}} \mathbf{R}[\mathbf{x}]_{\mathcal{R}}
$$

whereas the Hessian yields

$$
\frac{\partial^{2} \bar{V}}{\partial[\mathbf{v}]_{\mathcal{F}}^{2}}=\mathbf{B}
$$

One may readily verify, from (17), that $\mathbf{B}$ is symmetric positivedefinite, and therefore, all stationary points $\mathbf{v}$ of $\bar{V}$ are minima. Matrix $\mathbf{B}$ being nonsingular, $\partial \bar{V} / \partial[\mathbf{v}]_{\mathcal{F}}$ of (25), admits one single root, namely,

$$
[\mathbf{v}]_{\mathcal{F}}=\mathbf{B}^{-1} \mathbf{A}^{\mathrm{T}} \mathbf{R}[\mathbf{x}]_{\mathcal{R}} .
$$

Upon substituting (21) into the foregoing equation, we obtain

$$
[\mathbf{w}]_{\mathcal{R}}=\mathbf{K}[\mathbf{x}]_{\mathcal{R}}, \quad \text { where } \mathbf{K} \equiv \mathbf{R}^{\mathrm{T}} \mathbf{A B}^{-1} \mathbf{A}^{\mathrm{T}} \mathbf{R} .
$$

The potential energy can now be written as a function of the system posture $\mathbf{x}$, namely,

$$
V=(1 / 2) \mathbf{v}^{\mathrm{T}} \mathbf{K} \mathbf{x} .
$$

\section{Dissipated Energy}

Damping in MEMS is known to be the result of a variety of phenomena (e.g., air damping, clamp losses, thermoelastic 
dissipation, and crystallographic defects) that are generally nonlinear functions of the system posture $\mathbf{x}$ and its derivative $\dot{\mathbf{x}}$. However, for small displacements $\mathbf{x}$ and a narrow bandwidth, it is often possible to linearize these functions around the operating point of the mechanical system. In the case of nonlinear damping, the resulting ordinary differential equation is not likely to admit a closed-form solution. In the analysis that follows, we decided to restrict ourselves to applications where observable-damping phenomena may be linearized around the operating point of the system. Moreover, it is assumed that damping acts on the rigid links alone, which allows us to define the Rayleigh dissipation function

$$
P=(1 / 2) \dot{\mathbf{x}}^{\mathrm{T}} \mathbf{C} \dot{\mathbf{x}}
$$

where $\mathbf{C} \in \mathbb{R}^{n \times n}$ is, at least, positive-semidefinite and contains the system damping coefficients.

\section{E. Dynamic Model of the Compliant Mechanism}

The Lagrangian of the mechanism is readily computed as

$$
L \equiv T-V=(1 / 2) \dot{\mathbf{x}}^{\mathrm{T}} \mathbf{M} \dot{\mathbf{x}}-(1 / 2) \mathbf{x}^{\mathrm{T}} \mathbf{K} \mathbf{x}
$$

and its associated Lagrange equations are

$$
\frac{d}{d t}\left(\frac{\partial L}{\partial \dot{\mathbf{x}}}\right)-\frac{\partial L}{\partial \mathbf{x}}=-\frac{\partial P}{\partial \dot{\mathbf{x}}}+\mathbf{w}
$$

where

$$
\mathbf{M} \ddot{\mathbf{x}}+\mathbf{K x}=-\mathbf{C} \dot{\mathbf{x}}+\mathbf{w}
$$

which is the mathematical model sought. As the mass matrix is bound to be symmetric and positive-definite, we can compute its Cholesky decomposition as $\mathbf{M}=\mathbf{L L}^{\mathrm{T}}$. This allows us to rewrite (33) in its monic representation [22] by performing the change of variable $\mathbf{z}=\mathbf{L}^{\mathrm{T}} \mathbf{x}$, namely,

$$
\ddot{\mathbf{z}}+\Delta \dot{\mathbf{z}}+\Omega^{2} \mathbf{z}=\mathbf{L}^{-1} \mathbf{w}
$$

where $\boldsymbol{\Delta} \equiv \mathbf{L}^{-1} \mathbf{C} \mathbf{L}^{-\mathrm{T}}$ is the dissipation matrix and $\boldsymbol{\Omega}^{2} \equiv$ $\mathbf{L}^{-1} \mathbf{K} \mathbf{L}^{-\mathrm{T}}$ is the square of the frequency matrix of the undamped compliant mechanism.

Let $\mu_{i}$ and $\boldsymbol{\mu}_{i}, i=1, \ldots, n$, be the eigenvalues and eigenvectors of $\Omega^{2}$, respectively; the former being identical to the natural frequencies squared, the latter linear transformations of the modal vectors of the undamped system of (34). That is, if we let $\lambda_{i}$ and $\lambda_{i}$ be the natural frequencies and the modal vectors of the undamped nonexcited system [(33)], then

$$
\begin{aligned}
\lambda_{i} & =\sqrt{\mu_{i}} \\
\boldsymbol{\lambda}_{i} & =\mathbf{L}^{-\mathrm{T}} \boldsymbol{\mu}_{i}, \quad i=1, \ldots, n .
\end{aligned}
$$

Let us pursue this analysis by computing the Laplace-domain transfer function $\mathbf{H}(s)$, which maps the input wrenches $\mathbf{w}$ acting on the rigid bodies onto the system states $\mathbf{x}$, and where $s$ is the variable of the Laplace domain. From (33), we have

$$
\mathbf{H}(s)=\mathbf{L}^{-\mathrm{T}}\left(\mathbf{1}_{6 n \times 6 n} s^{2}+\Delta s+\boldsymbol{\Omega}^{2}\right)^{-1} \mathbf{L}^{-1} .
$$

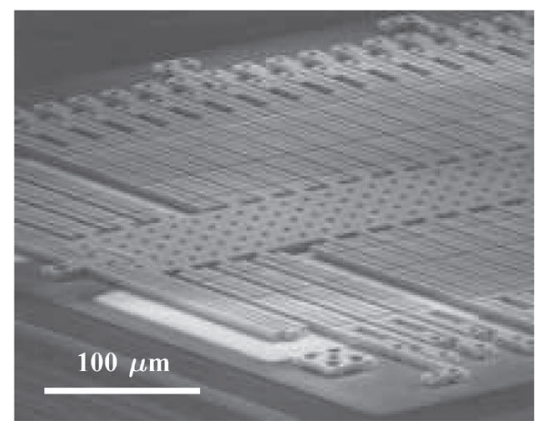

Fig. 3. Analog Devices ADXL150.

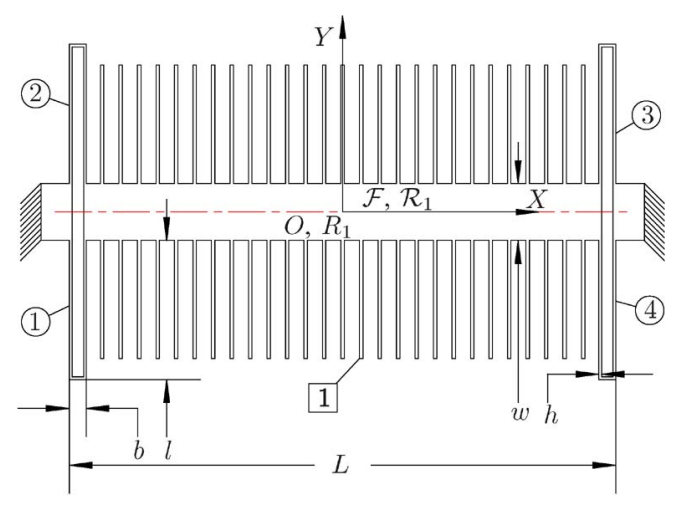

Fig. 4. Mechanical structure of ADXI 150.

In the balance of this paper, the proposed mathematical model is applied to two MEMS devices. The ensuing estimates of the dynamic properties are then compared against those obtained from other methods.

\section{Analysis of a Simple Case: The ADXL150 ACCELERometer From ANALOg DeVices}

Even though it is now replaced with the ADXL78, the ADXL150 accelerometer from Analog Devices has been a reference for accelerometer designers [18]. Because of that, its properties are known, and it is thus a good starting point to validate the proposed mathematical model and to show its usefulness. The ADXL150 has a range of action of $\pm 50 \mathrm{~g}$. It is fabricated using surface-micromachining techniques, which allows for a size as small as $753 \times 657 \mu \mathrm{m}$, which can be appreciated from Fig. 3.

The ADXL150 is a uniaxial accelerometer, and hence, its stiffness should be much lower along its sensitive axis than along any other direction. To verify this, we analyze the mechanical structure of the device, which is shown in Fig. 4. In this sketch, the thickness $t$ of the compliant mechanism is measured in the direction orthogonal to the plane of the figure. The compliant legs are numbered in encircled numerals from one to four, whereas the only rigid link of this mechanism is the proof mass itself, which is thus labeled with number one, in a square.

The dimensions are recorded in Table I, as taken from [18], except for $b$, which was estimated from Fig. 3. Frames $\mathcal{F}$ and $\mathcal{R}_{1}$ are defined as shown in Fig. 4, with their $X$-axes 
TABLE I

DIMENSIONS OF THE ADXL150 ACCELEROMETER

\begin{tabular}{|c|c|c|c|c|c|}
\hline$L$ & $l$ & $w$ & $b$ & $h$ & $t$ \\
$500 \mu \mathrm{m}$ & $120 \mu \mathrm{m}$ & $50 \mu \mathrm{m}$ & $10 \mu \mathrm{m}$ & $2.5 \mu \mathrm{m}$ & $2 \mu \mathrm{m}$ \\
\hline
\end{tabular}

TABLE II

SCREWS $\mathbf{s}_{i}\left(s_{i}\right), i=1,2,3,4$

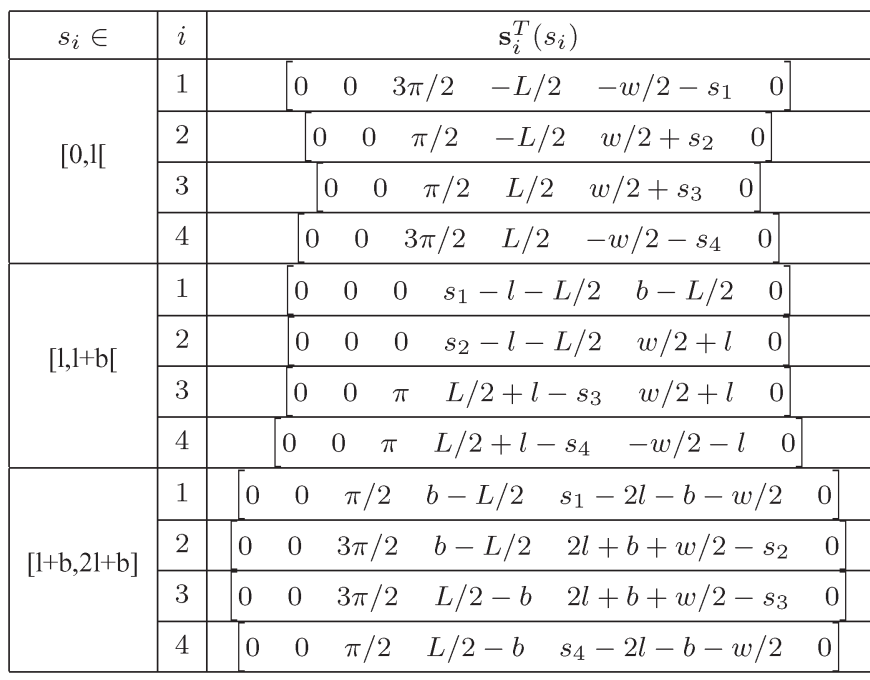

along the accelerometer sensitive axis-represented here by a dashed line-and with their $Y$-axes in the plane of the wafer surface. We take those frames to be right-handed, and so, their $Z$-axes stem out of the plane of the wafer surface. Moreover, the origins $O$ and $R_{1}$ of these two frames are located at the proofmass centroid. Hence, apparently, frames $\mathcal{F}$ and $\mathcal{R}_{j}$ are chosen to be identical, which simplifies subsequent calculations. The material of the flexible mechanism is polysilicon, which has a Young modulus $E=160 \mathrm{GPa}$, a Poisson ratio $\nu=0.2$, and a density $\rho=2331 \mathrm{~kg} / \mathrm{m}^{3}$. The mass matrix $\mathbf{M}$ of the mechanism is the mass matrix of the proof mass as defined in (6). The corresponding inertia matrix is estimated to be

$$
\left[\mathbf{I}_{1}\right]_{\mathcal{F}}=\left[\mathbf{I}_{1}\right]_{\mathcal{R}, 1}=\left[\begin{array}{ccc}
0.5586 & 0 & 0 \\
0 & 3.088 & 0 \\
0 & 0 & 3.647
\end{array}\right] \times 10^{-18} \mathrm{~kg} \cdot \mathrm{m}^{2}
$$

from a computer-aided-design (CAD) model of the proof mass that included its 54 electrodes, whereas the mass is taken to be $m_{1}=2.2 \times 10^{-10} \mathrm{~kg}$, the same value as that used by [18]. This allows the evaluation of the kinetic energy from (7).

On the other hand, calculating the potential energy requires the definition of the additional frames $\mathcal{S}_{i}\left(s_{i}\right), i=1,2,3,4$. This can be done through the definition of their associated screws $\mathbf{s}_{i}\left(s_{i}\right), i=1,2,3,4$, which take frame $\mathcal{R}_{1}$ into their respective frames $\mathcal{S}_{i}\left(s_{i}\right), i=1,2,3,4$. Because the compliant links exhibit discontinuous neutral axes, these screws are defined as piecewise functions, as detailed in Table II.

The beam cross section remains constant in all the compliant links, and therefore, from the numerical data of Table II,
TABLE III

Modal ANALysis of THE ADXL150 ACCELERometer

\begin{tabular}{|c|c|c|c|c|c|c|}
\hline$i$ & 1 & 2 & 3 & 4 & 5 & 6 \\
\hline$f_{i}(\mathrm{kHz})$ & 19.11 & 24.38 & 36.08 & 39.90 & 175.60 & 334.62 \\
\hline \hline \multirow{6}{*}{$\boldsymbol{\lambda}_{i}$} & 0.000 & 0.000 & 1.000 & 0.000 & 0.000 & 0.000 \\
& 0.000 & 0.000 & 0.000 & 1.000 & 0.000 & 0.000 \\
& 0.000 & 0.000 & 0.000 & 0.000 & 0.000 & 1.000 \\
& 0.000 & 1.000 & 0.000 & 0.000 & 0.000 & 0.000 \\
& 0.000 & 0.000 & 0.000 & 0.000 & 1.000 & 0.000 \\
& 1.000 & 0.000 & 0.000 & 0.000 & 0.000 & 0.000 \\
\hline
\end{tabular}

we obtain

$$
\begin{aligned}
J_{i} & =\beta h t^{3}=3.37 \times 10^{-24} \mathrm{~m}^{4}, \quad \text { with } \quad \beta=0.1685 \\
I_{Y, i} & =t^{3} h / 12=1.67 \times 10^{-24} \mathrm{~m}^{4} \\
I_{Z, i} & =h^{3} t / 12=2.60 \times 10^{-24} \mathrm{~m}^{4} \\
A_{i} & =h t=5.00 \times 10^{-12} \mathrm{~m}^{2} \\
\alpha_{Y, i} & =\alpha_{Z, i}=6 / 5 .
\end{aligned}
$$

Since frames $\mathcal{F}$ and $\mathcal{R}_{1}$ are coincident, we have

$$
\mathbf{R}=\mathbf{R}_{1}=1_{6 \times 6}
$$

and because the four compliant links connect the fixed rigid link to the only mobile rigid link, we may assign

$$
\mathbf{A}=\left[\begin{array}{llll}
-1_{6 \times 6} & -1_{6 \times 6} & -1_{6 \times 6} & -1_{6 \times 6}
\end{array}\right]
$$

where the minus sign comes from the assumption that the fixed rigid link corresponds to the index $j=0$. From (28), we obtain directly

$$
\begin{aligned}
\mathbf{K}=\operatorname{diag}\left(2.87 \times 10^{-8},\right. & 1.94 \times 10^{-7} \\
& \left.1.61 \times 10^{-5}, 5.16,268,3.17\right)
\end{aligned}
$$

whose first three entries bear units of newton meters per radian, the last three of newtons per meter. The fourth diagonal term in (41) represents the stiffness of the mechanism along the accelerometer sensitive axis. The value reported in [13] for the same dimensions was $5.6 \mathrm{~N} / \mathrm{m}$, but this did neither take into account the deflection of the shorter intermediate straight beams in each leg, i.e., the beams with a length of $b=10 \mu \mathrm{m}$, nor the shear strain in any of the beams. According to [18], the actual value measured by Analog Devices is $5.4 \mathrm{~N} / \mathrm{m}$. Hence, in this paper, the proposed model appears to be accurate.

The frequency matrix can thus be computed from its definition, in (34), which yields

$$
\begin{aligned}
& \Omega=\operatorname{diag}(2.272 .5121 .01 .6712 .01 .31) \times 10^{5} \mathrm{rad} / \mathrm{s} \\
& =\operatorname{diag}(36.1 \quad 39.9 \quad 335 \quad \mathbf{2 4 . 4} \quad \mathbf{1 7 6} \quad \mathbf{1 9 . 1}) \mathrm{kHz} .
\end{aligned}
$$

Apparently, the frequency matrix is diagonal, which allows for the extraction of its eigenvalues and eigenvectors by simple inspection. For the sake of clarity, these values are listed in Table III, in ascending order.

A natural frequency of $24.7 \mathrm{kHz}$ is reported in [18] in the direction of the accelerometer sensitive axis, which is somewhat larger than the $24.4 \mathrm{kHz}$ obtained here. The reason behind this 
is the different stiffness estimate which, in the case of [18], did not take into account the shear strain nor the compliance of the intermediate beam in each leg of the ADXL150. There is a relatively large difference between the fundamental frequency and that of the mode in the direction of the accelerometer sensitive axis, as compared to the small difference of the former with that of a translational out-of-plane motion of the proof mass, which may surprise anyone who is not familiar with MEMS design. Indeed, at this scale, the damping due to the squeezed film of air between the proof mass and the substrate is extremely high in that direction, which prevents this parasitic motion from becoming significant at high frequencies.

To substantiate this claim, let us estimate the damping in the system by assigning some values to matrix $\mathbf{C}$ defined in (30). Here, we consider only the damping that is due to air friction beneath the proof mass and between the electrodes. Moreover, even though it may be just as high as the damping due to proof-mass translations, we neglect air damping due to proof-mass rotations. This choice is mainly justified by virtue of correspondence of the lowest frequencies of the system to those of the translational proof-mass motions; the choice can also be justified by the level of complexity associated with the modeling of the air flow around a rotating plate close to a flat surface. Indeed, the air flow beneath the proof mass induced by in-wafer-plane translations may be modeled as a simple Couette ${ }^{4}$ flow, whereas the air flow produced by outof-wafer-plane translations may be assimilated to a Poiseuille ${ }^{5}$ flow [18]. Moreover, as a rough approximation, we consider the flow between the electrodes as well as when the proof mass translates in the $X$-axis direction to be of the Poiseuille type; we consider a Couette flow when the proof mass translates in the $Y$-axis direction. As a result, we have

$$
\begin{gathered}
\mathbf{C}=\operatorname{diag}\left\{0,0,0, \eta(L-2 b) w / c+108 \times 96 \eta l t^{3} /\left(\pi^{4} d^{3}\right),\right. \\
\left.\eta(L-2 b) w / c+108 \eta l t / d, 96 \eta(L-2 b) w^{3} /\left(\pi^{4} c^{3}\right)\right\}
\end{gathered}
$$

i.e.,

$$
\begin{aligned}
\mathbf{C}=\operatorname{diag}\left\{0,0,0,1.107 \times 10^{-6},\right. \\
\left.0.629 \times 10^{-6}, 259.858 \times 10^{-6}\right\} \mathrm{N} \cdot \mathrm{s} / \mathrm{m}
\end{aligned}
$$

where $c=1.6 \mu \mathrm{m}$ is the gap between the proof mass and the chip, $d=1.3 \mu \mathrm{m}$ is the gap between two electrodes, and $\eta=18 \times 10^{-6} \mathrm{~Pa} \cdot \mathrm{s}$ is the dynamic viscosity of air.

The associated matrix transfer function is computed from (36). In the case of an accelerometer, the array of rigid-link external wrenches $\mathbf{w}$ defined in (20) may be regarded, from d'Alembert's principle, as an array of inertial wrenches. Moreover, if we assume that the instant screw axis of any motion of the accelerometer frame lies at infinity, which is reasonable for a small mechanical system, we can neglect the angular velocity and write $\mathbf{w}$ as a linear function of the twist timederivative $\mathbf{a}$ of the accelerometer frame. Hence, the acceleration

\footnotetext{
${ }^{4} \mathrm{~A}$ Couette flow is a laminar flow of an incompressible Newtonian fluid induced by the relative motion of the two parallel planes in relative translation that contain the fluid [23].

${ }^{5}$ A Poiseuille flow is a laminar flow of an incompressible Newtonian fluid in a pipe [23].
}

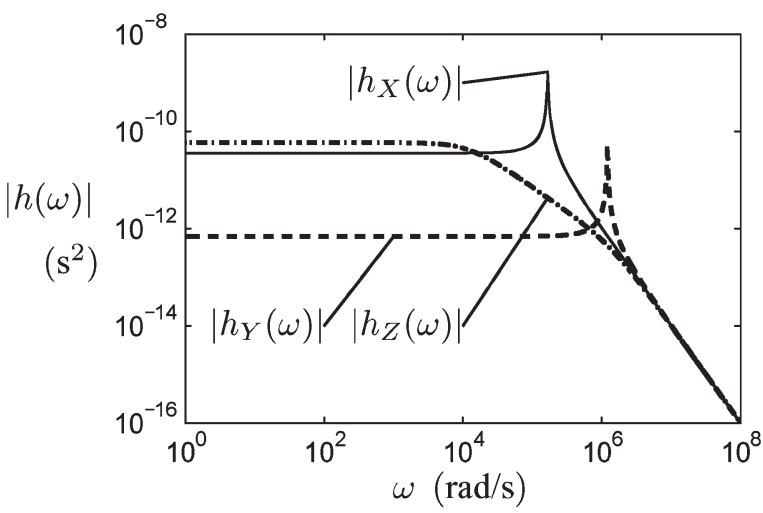

(a)

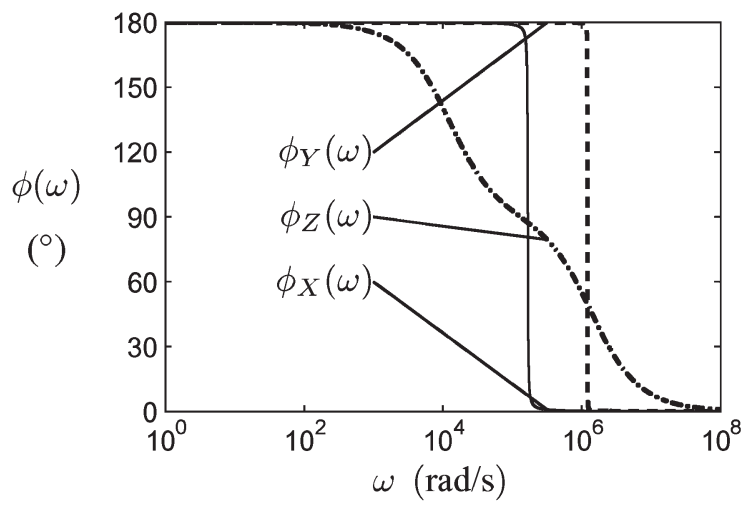

(b)

Fig. 5. Frequency response of the ADXL150. (a) Magnitude. (b) Phase.

field of the accelerometer frame is approximated by a helical field represented by screw $\mathbf{a}$, which is formed with the angular acceleration of frame $\mathcal{F}$ with respect to an inertial frame, and the acceleration of its origin $O$ with respect to a fixed reference point, both expressed in the accelerated frame $\mathcal{F}$. Symbolically, we obtain

$$
\mathbf{w}=-\mathbf{M R}^{\mathrm{T}} \mathbf{T a}
$$

where $\mathbf{T} \equiv\left[\begin{array}{lll}\mathbf{1}_{6 \times 6} & \mathbf{1}_{6 \times 6} \cdots \mathbf{1}_{6 \times 6}\end{array}\right]^{\mathrm{T}}$.

Let us now label $\xi_{X}, \xi_{Y}$, and $\xi_{Z}$, the components of the position of the proof-mass position vector $\boldsymbol{\xi}_{1}$, and $a_{X}, a_{Y}$, and $a_{Z}$, the components of $\mathbf{a}$. We also define unit vectors $\mathbf{e}_{4} \equiv\left[\begin{array}{lll}\mathbf{0}_{3}^{\mathrm{T}} & 1 & \mathbf{0}_{2}^{\mathrm{T}}\end{array}\right]^{\mathrm{T}}, \mathbf{e}_{5} \equiv\left[\begin{array}{lll}\mathbf{0}_{4}^{\mathrm{T}} & 1 & 0\end{array}\right]^{\mathrm{T}}$, and $\mathbf{e}_{6} \equiv\left[\begin{array}{ll}\mathbf{0}_{5}^{\mathrm{T}} & 1\end{array}\right]^{\mathrm{T}}$, which lets us write the input-output relationships

$$
\begin{gathered}
h_{X}(s) \equiv \xi_{X}(s) / a_{X}(s)=-\mathbf{e}_{4}^{\mathrm{T}} \mathbf{H}(s) \mathbf{M} \mathbf{R}^{\mathrm{T}} \mathbf{T} \mathbf{e}_{4} \\
h_{Y}(s) \equiv \xi_{Y}(s) / a_{Y}(s)=-\mathbf{e}_{5}^{\mathrm{T}} \mathbf{H}(s) \mathbf{M} \mathbf{R}^{\mathrm{T}} \mathbf{T e}_{5} \\
h_{Z}(s) \equiv \xi_{Z}(s) / a_{Z}(s)=-\mathbf{e}_{6}^{\mathrm{T}} \mathbf{H}(s) \mathbf{M} \mathbf{R}^{\mathrm{T}} \mathbf{T e}_{6} .
\end{gathered}
$$

We compute the complex frequency responses $h_{X}(\omega), h_{Y}(\omega)$, and $h_{Z}(\omega)$ of the proof mass to transverse accelerations by evaluating the corresponding transfer functions at $\omega \sqrt{-1}$, where $\omega$ is the input frequency. Upon computing the magnitudes and phase angles of these complex functions, we obtain the Bode plots shown in Fig. 5.

From the magnitude-versus-frequency plot shown in Fig. 5(a), we see that the accelerometer response to $Z$-axis accelerations is overdamped, whereas the responses to in-wafer 


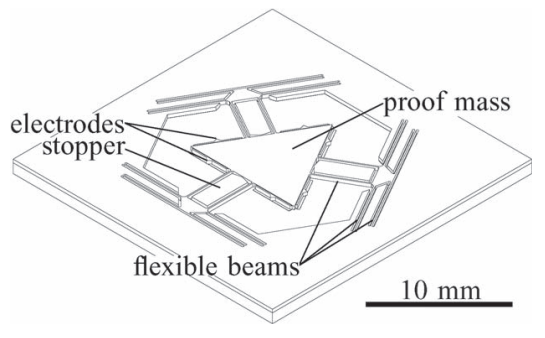

Fig. 6. CAD model of the SBA.

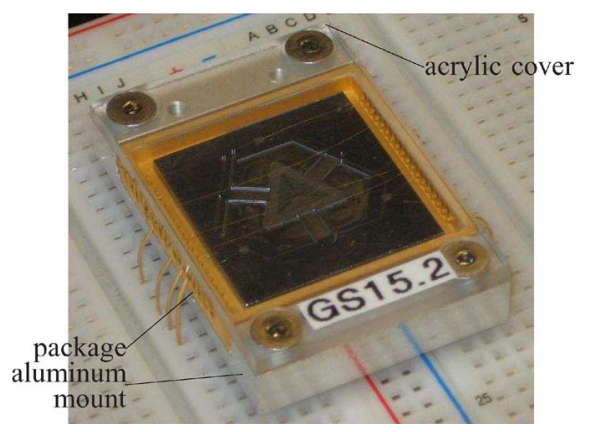

Fig. 7. Package microfabricated SBA.

plane accelerations are underdamped. Nevertheless, the outof-wafer-plane motion can still pose problems when the proof mass is subjected to low-frequency accelerations (e.g., gravitational or centripetal) along the $Z$-direction of frame $\mathcal{F}$. Indeed, cross-axis sensitivity is the highest source of errors $( \pm 2 \%$ of the full-scale range, i.e., $1 g$ ) for the ADXL150 accelerometer.

\section{Analysis of a Complex Case: The SBA}

Let us now turn our attention toward a more complex mechanical architecture, that of the simplicial biaxial accelerometer (SBA) [24], which is shown in Fig. 6. The proof mass of this accelerometer takes the shape of a regular triangle. This triangle is suspended at each of its edges by a pair of distal beams, which connects at its other ends to an intermediate rigid link. In turn, this rigid link is suspended by four proximal beams perpendicular to their corresponding pair of distal beams, which connect to the accelerometer frame at their other ends. As a result, this mechanism is compliant to proof-mass translations in the wafer plane, while offering high stiffness to proof-mass rotations and translations out of the wafer plane. The equilateral triangular geometry of the SBA resembles that of the HexFlex [25] while resulting in a completely different mechanical behavior. If the SBA allows for in-plane translations of its moving platform, the HexFlex allows for any displacements of its moving platform in space. Moreover, in the case of the SBA, the moving platform serves as an acceleration sensor, whereas the HexFlex moving platform is actuated through its supporting legs. A prototype of the packaged microfabricated device is shown in Fig. 7.

For the purpose of this analysis, a top view of the SBA is shown in Fig. 8, where each link is labeled with a number appearing in a circle for a compliant link and in a box for a rigid link. Table IV lists the numerical values of the dimensions shown in Fig. 8 as well as the numerical value of the device wafer thickness $t$.

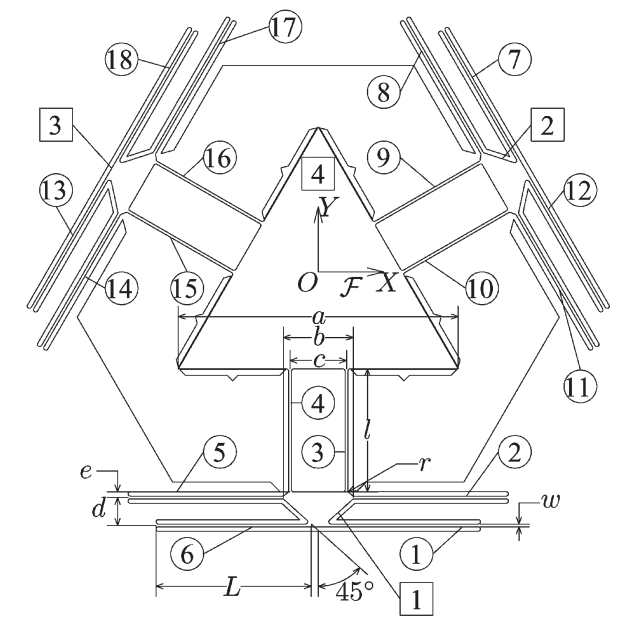

Fig. 8. Dimensions of the SBA.

TABLE IV

DIMENSIONS OF THE SBA

\begin{tabular}{|c|c|c|c|c|}
\hline$L$ & $l$ & $w$ & $t$ & $r$ \\
\hline $5544 \mu \mathrm{m}$ & $4400 \mu \mathrm{m}$ & $105 \mu \mathrm{m}$ & $300 \mu \mathrm{m}$ & $78.7 \mu \mathrm{m}$ \\
\hline$a$ & $b$ & $c$ & $d$ & $e$ \\
\hline $10000 \mu \mathrm{m}$ & $2500 \mu \mathrm{m}$ & $2020 \mu \mathrm{m}$ & $1000 \mu \mathrm{m}$ & $200 \mu \mathrm{m}$ \\
\hline
\end{tabular}

\section{A. Kinetic Energy}

Frames $\mathcal{R}_{j}, j=1, \ldots, n, n=4$, are located at the mass centers of their corresponding rigid links and oriented so that screws $\mathbf{r}_{j}, j=1, \ldots, n$, take the values as shown at the bottom of the next page, where

$$
f \equiv \frac{(b+c)\left(b e^{2}-2 d^{3}+3 b d^{2}+6 b d e-6 d e^{2}\right)-2 e^{2} c^{2}}{3(b+c)(e(b-c)+2 d(b-d))} .
$$

The mass properties of the rigid links are computed from a CAD model, which yields

$$
\begin{aligned}
& m_{1}=m_{2}=m_{3}=1.387 \times 10^{-3} \mathrm{~kg} \\
& m_{4}=30.25 \times 10^{-3} \mathrm{~kg} \\
& {\left[\mathbf{I}_{1}\right]_{\mathcal{R}}, 1=\left[\mathbf{I}_{2}\right]_{\mathcal{R}, 2}=\left[\mathbf{I}_{3}\right]_{\mathcal{R}, 3}} \\
& =\operatorname{diag}\left(\begin{array}{lll}
0.1456 & 0.4656 & 0.5904
\end{array}\right) \times 10^{-9} \mathrm{~kg} \cdot \mathrm{m}^{2} \\
& {\left[\mathbf{I}_{4}\right]_{\mathcal{R}, 4}=\operatorname{diag}(12.63 \quad 12.63 \quad 252.1) \times 10^{-9} \mathrm{~kg} \cdot \mathrm{m}^{2} .}
\end{aligned}
$$

The mass matrix is evaluated directly from these numerical values and the definition of (7).

\section{B. Potential Energy}

Upon defining the lengths $g \equiv b / 2+L$ and $h \equiv \sqrt{3} a / 6+$ $l+e$, screws $\mathbf{s}_{i}, i=1, \ldots, m, m=18$, are evaluated as the second set of equations shown at the bottom of the next page.

Turning our attention to the elastostatic properties of the compliant links, we realize that the beams are not all identical in that respect, since silicon crystal is an anisotropic material. The axes of the cubic crystal correspond to the axes of frame $\mathcal{F}$ shown in Fig. 8. As a result, the Young moduli $E_{i}$ and the shear moduli $G_{i}, i=1, \ldots, m$, are not all equal, depending on the orientation of their corresponding compliant link with respect 
to the crystal axes. Because of the symmetry in the crystal, we have

$$
\mathbf{H}_{i}=\operatorname{diag}\left(\frac{1}{G_{\perp} J}, \frac{1}{E_{\perp} I_{Y}}, \frac{1}{E_{\perp} I_{Z}}, \frac{1}{E_{\perp} A}, \frac{\alpha_{Y}}{G_{\perp} A}, \frac{\alpha_{Z}}{G_{\perp} A}\right)
$$

for $i=1, \ldots, 6$, and

$$
\mathbf{H}_{i}=\operatorname{diag}\left(\frac{1}{G_{\angle, T} J}, \frac{1}{E_{\angle} I_{Y}}, \frac{1}{E_{\angle I} I_{Z}}, \frac{1}{E_{\angle A} A}, \frac{\alpha_{Y}}{G_{\angle, Y} A}, \frac{\alpha_{Z}}{G_{\angle, Z} A}\right)
$$

for $i=7, \ldots, 18$, where the $\perp$ subscript refers to the beams that are at $0^{\circ}$ or $90^{\circ}$ from a crystal axis, and the $\angle$ subscript refers to the beams that are at $30^{\circ}$ or $60^{\circ}$ from a crystal axis. Because of material anisotropy, two distinct shear moduli are associated with the cross sections that are not orthogonal to one of the crystallographic axes. These moduli, which we label $G_{\angle, Y}$ and $G_{\angle, Z}$, correspond, respectively, to the directions of the $Y_{\mathcal{S}, i^{-}}$and $Z_{\mathcal{S}, i^{-}}$axes of the cross-sectional frames $\mathcal{S}_{i}\left(s_{i}\right)$, $i=7, \ldots, 18$. Moreover, in this model, we neglect any coupling between torsion and bending due to the distinct shear moduli in compliant links $i=7, \ldots, 18$. In fact, to avoid overcomplicating the problem, we define the section shear modulus in torsion as the average of the two actual shear moduli of the section, i.e.,

$$
G_{\angle, T} \equiv\left(G_{\angle, Y}+G_{\angle, Z}\right) / 2 .
$$

This saves us from resorting to a modified Saint-Venant method, which would require the solution of a partial differential equation of the form

$$
G_{\llcorner, Y} \frac{\partial^{2} \phi(y, z)}{\partial y^{2}}+G_{\llcorner, Z} \frac{\partial^{2} \phi(y, z)}{\partial z^{2}}=0
$$

where $\phi(y, z)$ represents the warping of the beam cross sections. Hence, in the case of pure torsion, we consider the material to be isotropic with a shear modulus of $G_{\angle, T}$.

$$
\begin{aligned}
\mathbf{r}_{1} & =\left[\begin{array}{llll}
\mathbf{0}_{4}^{\mathrm{T}} & -\sqrt{3} a / 6-l-f & 0
\end{array}\right]^{\mathrm{T}} \\
\mathbf{r}_{2} & =\left[\begin{array}{lllll}
\mathbf{0}_{2}^{\mathrm{T}} & 2 \pi / 3 & (a / 2+\sqrt{3} l+\sqrt{3} f) / 2 & (\sqrt{3} a / 6+l+f) / 2 & 0
\end{array}\right]^{\mathrm{T}} \\
\mathbf{r}_{3} & =\left[\begin{array}{lllll}
\mathbf{0}_{2}^{\mathrm{T}} & -2 \pi / 3 & -(a / 2+\sqrt{3} l+\sqrt{3} f) / 2 & (\sqrt{3} a / 6+l+f) / 2 & 0
\end{array}\right]^{\mathrm{T}} \\
\mathbf{r}_{4} & =\mathbf{0}_{6}
\end{aligned}
$$

$$
\begin{aligned}
& \mathbf{s}_{1}=\left[\begin{array}{llllll}
0 & 0 & \pi & g-d-s_{1} & -h-d & 0
\end{array}\right]^{\mathrm{T}} \\
& \mathbf{s}_{2}=\left[\begin{array}{llllll}
0 & 0 & \pi & g-s_{2} & -h & 0
\end{array}\right]^{\mathrm{T}} \\
& \mathbf{s}_{3}=\left[\begin{array}{llllll}
0 & 0 & \pi / 2 & c / 2 & s_{3}-\sqrt{3} a / 6-l & 0
\end{array}\right]^{\mathrm{T}}=\mathbf{s}_{9}=\mathbf{s}_{15} \\
& \mathbf{s}_{4}=\left[\begin{array}{llllll}
0 & 0 & \pi / 2 & -c / 2 & s_{4}-\sqrt{3} a / 6-l & 0
\end{array}\right]^{\mathrm{T}}=\mathbf{s}_{10}=\mathbf{s}_{16} \\
& \mathbf{s}_{5}=\left[\begin{array}{llllll}
0 & 0 & 0 & s_{5}-g & -h & 0
\end{array}\right]^{\mathrm{T}} \\
& \mathbf{s}_{6}=\left[\begin{array}{llllll}
0 & 0 & 0 & s_{6}+d-g & -h-d & 0
\end{array}\right]^{\mathrm{T}}
\end{aligned}
$$

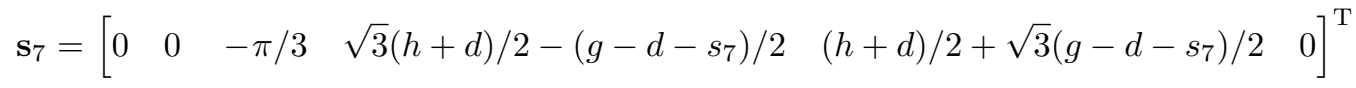

$$
\begin{aligned}
& \mathbf{s}_{8}=\left[\begin{array}{llllll}
0 & 0 & -\pi / 3 & \sqrt{3} h / 2-\left(g-s_{8}\right) / 2 & h / 2+\sqrt{3}\left(g-s_{8}\right) / 2 & 0
\end{array}\right]^{\mathrm{T}} \\
& \mathbf{s}_{11}=\left[\begin{array}{llllll}
0 & 0 & 2 \pi / 3 & \sqrt{3} h / 2+\left(g-s_{11}\right) / 2 & h / 2-\sqrt{3}\left(g-s_{11}\right) / 2 & 0
\end{array}\right]^{\mathrm{T}}
\end{aligned}
$$

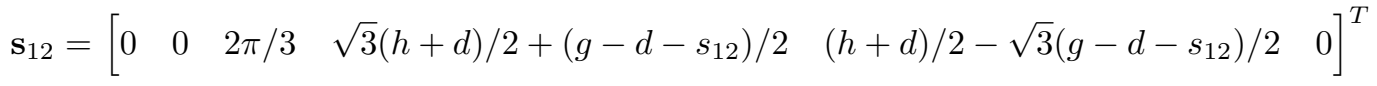

$$
\begin{aligned}
& \mathbf{s}_{13}=\left[\begin{array}{llllll}
0 & 0 & \pi / 3 & -\sqrt{3}(h+d) / 2-\left(g-d-s_{13}\right) / 2 & (h+d) / 2-\sqrt{3}\left(g-d-s_{13}\right) / 2 & 0
\end{array}\right]^{\mathrm{T}} \\
& \mathbf{s}_{14}=\left[\begin{array}{llllll}
0 & 0 & \pi / 3 & -\sqrt{3} h / 2-\left(g-s_{14}\right) / 2 & h / 2-\sqrt{3}\left(g-s_{14}\right) / 2 & 0
\end{array}\right]^{\mathrm{T}} \\
& \mathbf{s}_{17}=\left[\begin{array}{llllll}
0 & 0 & -2 \pi / 3 & -\sqrt{3} h / 2+\left(g-s_{17}\right) / 2 & h / 2+\sqrt{3}\left(g-s_{17}\right) / 2 & 0
\end{array}\right]^{\mathrm{T}}
\end{aligned}
$$

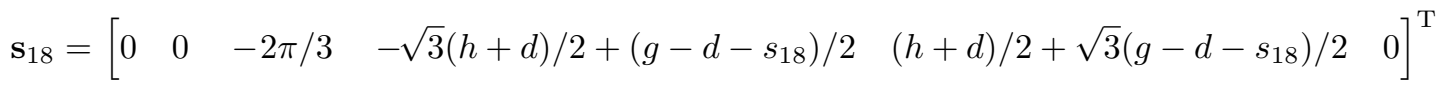


TABLE V

Cross-Sectional Properties of the COMPLIANT LINKS OF THE SBA

\begin{tabular}{|c|c|c|c|}
\hline$E_{\perp}$ & $130.2 \mathrm{GPa}$ & $G_{\perp}$ & $79.4 \mathrm{GPa}$ \\
\hline$E_{\llcorner, X}$ & $157.2 \mathrm{GPa}$ & $G_{\llcorner, Y}$ & $79.4 \mathrm{GPa}$ \\
\hline$G_{\llcorner, Z}$ & $55.9 \mathrm{GPa}$ & $G_{\llcorner, T}$ & $67.6 \mathrm{GPa}$ \\
\hline$J$ & $0.8965 \times 10^{-16} \mathrm{~m}^{4}$ & $I_{Y}$ & $0.2363 \times 10^{-15} \mathrm{~m}^{4}$ \\
\hline$I_{Z}$ & $0.2894 \times 10^{-16} \mathrm{~m}^{4}$ & $A$ & $0.3150 \times 10^{-7} \mathrm{~m}^{2}$ \\
\hline$\alpha_{Y}$ & 1.2 & $\alpha_{Z}$ & 1.2 \\
\hline
\end{tabular}

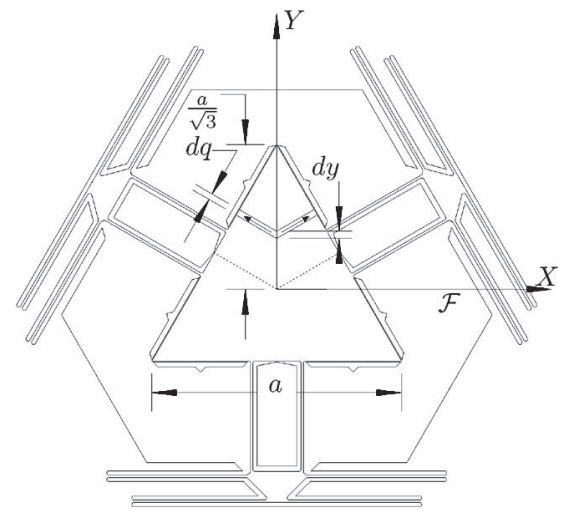

Fig. 9. Squeezed-film damping of the SBA proof mass.

The numerical values of the foregoing elastic properties are given in Table V, as reported in [26]. The geometric properties of the beam cross sections, also gathered in Table V, were computed from the formulas $J=\beta w t^{3}$, with $\beta=0.258$, $I_{Y}=w t^{3} / 12, I_{Z}=w^{3} t / 12$, and $A=w t$, whereas the shear correction factors are $\alpha_{Y}=\alpha_{Z}=6 / 5$ for rectangular cross sections [27]. This permits the computation of the stiffness matrix as defined in (28).

\section{Dissipated Energy}

It is assumed that all energy dissipation comes from air friction within the $2.5-\mu \mathrm{m}$ gap between the proof mass and the handle wafer. Following the same method as in Section III, a Couette flow is assumed for in-wafer-plane proofmass translations, while the air flow produced by proof-mass rotations and other rigid-link displacements are neglected. As in Section III, we may yet assume that an out-of-wafer-plane proof-mass translation generates a Poiseuille flow beneath it, but the triangular geometry of the proof mass prevents us from using the solution for rectangular plates. In order to obtain a rough estimate, let us assume a Poiseuille airflow beneath the proof mass that is orthogonal to the closest triangle edges, as shown in Fig. 9. Consider now the small element of area underneath the proof mass that has a width $d q$ and a total length of $a / \sqrt{3}-y$, where $y$ is the coordinate of the element intersection with the $Y$-axis. We may assume that the damping due to this small element is given by the formula for rectangular plates that have one side much larger than the other [18], i.e.,

$$
d c_{Z}=96 \eta(d q)(a / \sqrt{3}-y)^{3} /\left(\pi^{4} p^{3}\right)
$$

where $p=2.5 \mu \mathrm{m}$ is the gap between the proof mass and the handle wafer. Projecting the element width $d q$ onto the $Y$-axis yields the relation $d q=\sqrt{3} d y / 2$, which, upon substitution in (52), allows for a summation over the upper branch of the triangular proof mass that leads to the integral

$$
\begin{aligned}
c_{Z} / 3 & =\frac{48 \sqrt{3} \eta}{\pi^{4} p^{3}} \int_{0}^{a / \sqrt{3}}(a / \sqrt{3}-y)^{3} d y \\
& =\frac{48 \sqrt{3} \eta}{\pi^{4} p^{3}} \int_{0}^{a / \sqrt{3}} y^{3} d y
\end{aligned}
$$

where

$$
c_{Z}=\frac{4 \sqrt{3} \eta a^{4}}{\pi^{4} p^{3}}
$$

thereby obtaining the desired result.

As the result, matrix $\mathbf{C}$ of (30) takes the symbolic and numeric values

$$
\begin{aligned}
\mathbf{C}= & \operatorname{diag}\left(\left[\begin{array}{llll}
\mathbf{0}_{21}^{\mathrm{T}} & \eta A_{4} / p & \eta A_{4} / p & 4 \sqrt{3} \eta a^{4} /\left(\pi^{4} p^{3}\right)
\end{array}\right]^{\mathrm{T}}\right) \\
\mathbf{C =} & \operatorname{diag}\left(\left[\begin{array}{llll}
\mathbf{0}_{21}^{\mathrm{T}} & 3.12 \times 10^{-4} & 3.12 \times 10^{-4} & 8.19 \times 10^{2}
\end{array}\right]^{\mathrm{T}}\right) \\
& \times \mathbf{N} \cdot \mathrm{s} / \mathrm{m} .
\end{aligned}
$$

\section{Estimated Dynamics}

The first modes of the SBA are computed from (35), which yields the results of Table VI. Because of the 24 DOF of the compliant mechanism, these results are somewhat more intricate. The first two frequencies differ only by round-off error, their associated modes involving motions of all four rigid links. For acceleration measurement, we are interested only in proof-mass motions, which are represented here by vector $\boldsymbol{\lambda}_{i, 4}$. Apparently, from Table VI, the first two modes correspond to in-wafer-plane motions of the proof mass, with, in one case, a parasitic in-wafer-plane rotation. This parasitic motion is not due to round-off errors but rather to silicon anisotropy. One must bear in mind, however, that the rotational component of $\boldsymbol{\lambda}_{i, 4}$ is expressed in radians, whereas the translational component is expressed in meters. Hence, for instance, a $100-\mu \mathrm{m}$ displacement of the proof mass along the direction of the first mode results in a parasitic rotation of $7.88 \mathrm{rad}$ or to relative position errors of the vertices of the proof mass of $0.788 \mu \mathrm{m}$. This result is thought to be acceptable; we may safely say that the fundamental frequency corresponds now to the two sensitive directions. In turn, the third natural frequency appears to be dominated by rotations, except for a small nonnull value at the $Z$-axis translational direction of the proof mass. In fact, mode $\boldsymbol{\lambda}_{3}$ may be visualized as a rotation of the proximal rigid links about their associated $X_{i}$-axes-which are parallel to their corresponding edge of the triangular proof mass-and a translation-for the most part-of the proof mass along the $Z$-axis. Hence, we see that the natural frequency of the parasitic out-of-wafer-plane motion is now higher than that of the sensitive axes, which is, apparently, an advantage of 
TABLE VI

Modal ANALysis of THE SBA

\begin{tabular}{|c|c|c|c|c|c|c|}
\hline$i$ & 1 & 2 & 3 & 4 & 5 & 6 \\
\hline$f_{i}(\mathrm{~Hz})$ & 53.21 & 53.22 & 69.02 & 178.74 & 187.28 & 423.05 \\
\hline \multirow{6}{*}{$\boldsymbol{\lambda}_{i, 1}$} & 0.000 & -0.000 & -0.601 & 0.564 & 0.000 & -0.998 \\
\hline & -0.000 & 0.000 & 0.000 & 0.000 & 0.058 & 0.000 \\
\hline & 0.214 & 0.000 & -0.000 & -0.000 & -0.000 & -0.000 \\
\hline & -0.000 & -0.000 & -0.000 & -0.000 & -0.000 & -0.000 \\
\hline & -0.000 & 0.486 & 0.000 & -0.000 & -0.000 & -0.000 \\
\hline & 0.000 & -0.000 & -0.000 & 0.001 & 0.000 & 0.003 \\
\hline \multirow{6}{*}{$\boldsymbol{\lambda}_{i, 2}$} & -0.000 & -0.000 & -0.564 & -0.311 & -0.511 & -0.028 \\
\hline & -0.000 & 0.000 & -0.003 & 0.047 & -0.027 & -0.003 \\
\hline & -0.455 & -0.452 & -0.000 & -0.000 & -0.000 & -0.000 \\
\hline & -0.000 & 0.000 & 0.000 & -0.000 & -0.000 & 0.000 \\
\hline & 0.360 & -0.243 & -0.000 & 0.000 & -0.000 & -0.000 \\
\hline & -0.000 & 0.000 & -0.000 & -0.000 & -0.001 & 0.000 \\
\hline \multirow{6}{*}{$\boldsymbol{\lambda}_{i, 3}$} & 0.000 & -0.000 & -0.564 & -0.311 & 0.511 & -0.028 \\
\hline & -0.000 & 0.000 & 0.003 & -0.047 & -0.027 & 0.003 \\
\hline & -0.455 & 0.452 & -0.000 & -0.000 & -0.000 & -0.000 \\
\hline & -0.000 & -0.000 & -0.000 & -0.000 & 0.000 & 0.000 \\
\hline & -0.360 & -0.243 & -0.000 & 0.000 & 0.000 & -0.000 \\
\hline & 0.000 & -0.000 & -0.000 & -0.000 & 0.001 & 0.000 \\
\hline \multirow{6}{*}{$\boldsymbol{\lambda}_{i, 4}$} & -0.000 & -0.000 & -0.040 & 0.695 & 0.000 & -0.050 \\
\hline & -0.000 & -0.000 & 0.000 & -0.000 & -0.688 & -0.000 \\
\hline & 0.328 & 0.000 & -0.000 & -0.000 & 0.000 & -0.000 \\
\hline & 0.416 & 0.000 & 0.000 & 0.000 & -0.000 & 0.000 \\
\hline & 0.000 & -0.486 & -0.000 & 0.000 & 0.000 & 0.000 \\
\hline & -0.000 & 0.000 & 0.003 & 0.000 & 0.000 & 0.000 \\
\hline
\end{tabular}

the SBA mechanical architecture over that of the ADXL150 accelerometer.

In order to evaluate the effect of damping over the accelerometer, matrix $\mathbf{H}(s)$ is computed according to (36). As in Section III, we apply d'Alembert's principle of inertia forces to the dynamic system, taking the inertia forces acting on the rigid links as input forces of (33), the outputs are the proofmass displacements. In particular, upon applying successively pure accelerations along the $X$-, $Y$-, and $Z$-axis directions, we obtain the complex frequency responses $h_{X}(\omega), h_{Y}(\omega)$, and $h_{Z}(\omega)$, respectively, of translations of the proof mass in each of these directions. The magnitudes and phase angles of these frequency responses are shown in the Bode plots of Fig. 10. The lower sensitivity of the proof-mass displacements to $Z$-axis accelerations than to the $X$ - and $Y$-axis accelerations is confirmed from Fig. 10(a). One may also observe a good isotropy for in-wafer-plane accelerations and a bandwidth of approximately $100 \mathrm{rad} / \mathrm{s}$.

\section{E. Measured Dynamics}

The mechanical structure of the SBA was tested by applying to it short impulse accelerations in the negative direction of its $Z$-axis, as shown in Fig. 8. The "free" resonations of the structure were then recorded using a vibrometer. A schematic

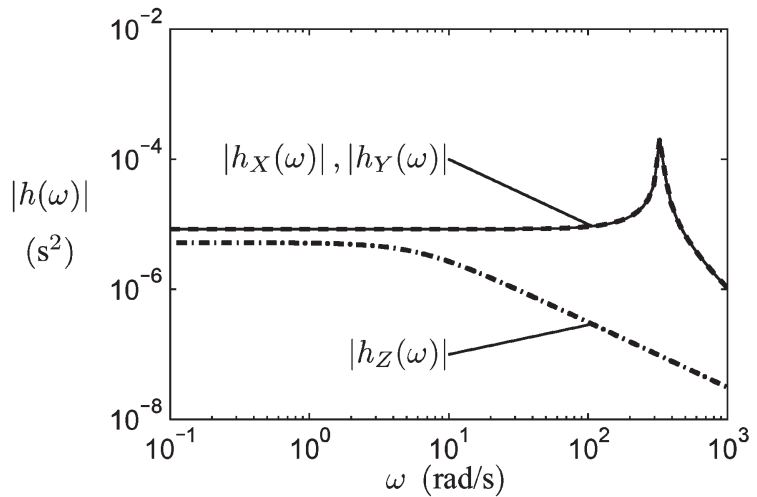

(a)

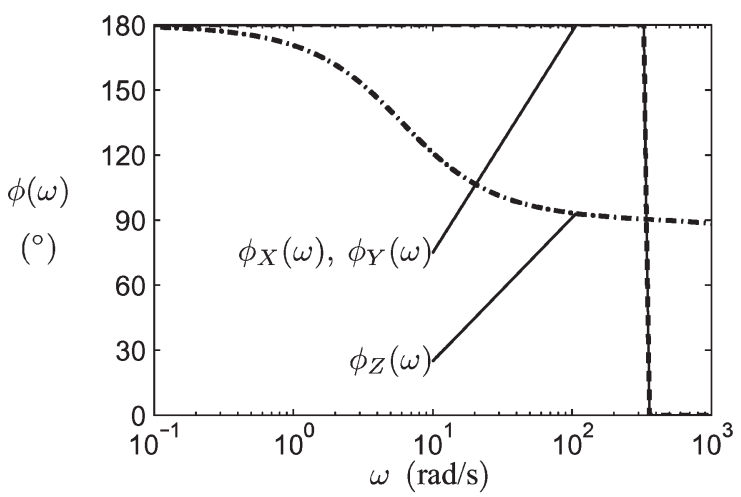

(b)

Fig. 10. Frequency response of the SBA. (a) Magnitude. (b) Phase angle.

and a picture of the test bench are shown in Fig. 11. In this setup, the shaker (Brüel and Kjaer Mini-Shaker 4810) is driven by a regular sound amplifier (Harman Kardon HK3300), which, itself, takes its input from a signal generator. A typical timehistory of the shaker impulse input voltage is shown in Fig. 12. The resulting motions of the SBA rigid links are recorded by the vibrometer (Polytec PSV-400), which sheds a laser beam vertically down on the sample. The laser beam is programmed to scan 382 points on the SBA, according to the mesh shown in Fig. 13. Point velocities are measured by the vibrometer, and thence, a frequency-domain distribution of the point velocities of the accelerometer architecture is computed and recorded by the controller (Polytec OFV-5000).

The Polytec controller returns a frequency-domain distribution of the velocities of the scanned points $\left\{S_{j}\right\}_{j=1}^{382}$, along with their phase correspondence with a reference signal. As shown in Fig. 11(a), in the test bench, the shaker input voltage was used as the reference signal. From these results, the complex velocity frequency response $v_{j}(\omega)$ of each point may be computed.

The point-displacement frequency response $d_{j}(\omega)$ may then be obtained as

$$
d_{j}(\omega)=v_{j} /(\omega i)
$$

where $i \equiv \sqrt{-1}$. On the other hand, point-acceleration frequency response $a_{j}(\omega)$ is given by

$$
a_{j}(\omega)=\omega i v_{j}
$$




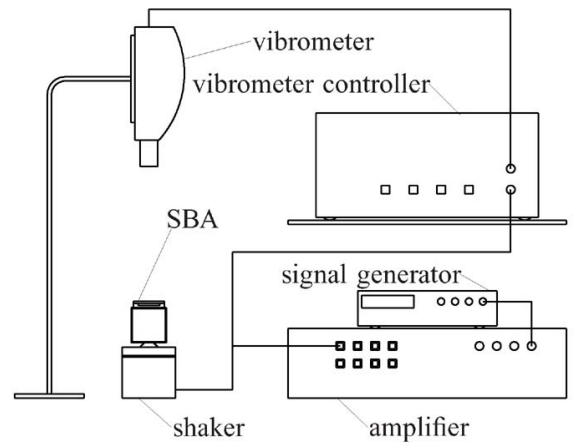

(a)

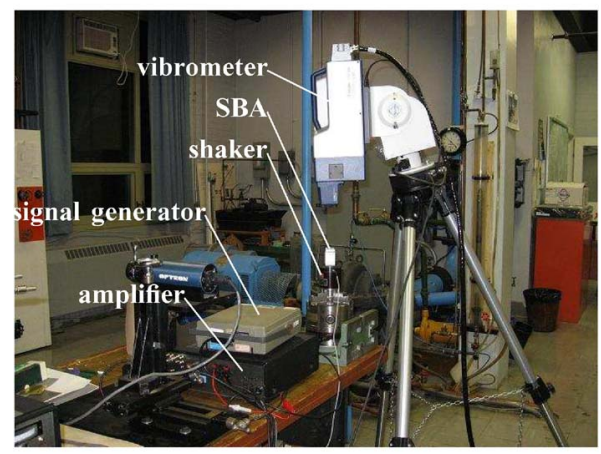

(b)

Fig. 11. SBA test bench. (a) Schematic representation. (b) Photograph.

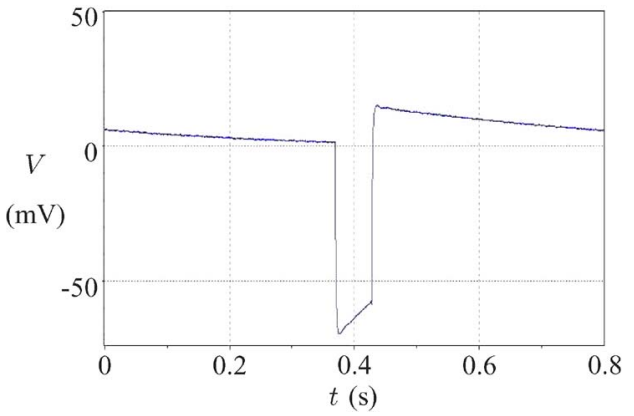

Fig. 12. Typical voltage impulse applied at the shaker input.

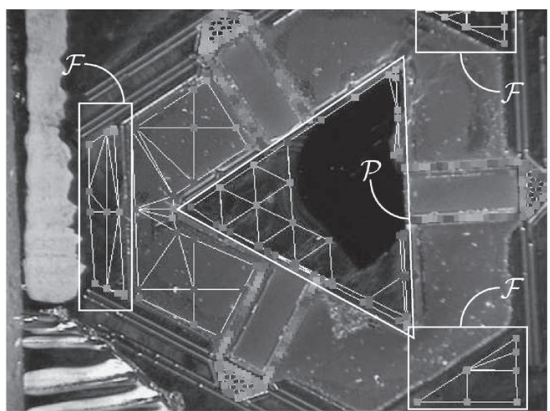

Fig. 13. Points scanned by the vibrometer.

Let us now define the sets $\mathcal{F}$ and $\mathcal{P}$ of the indexes of the points pertaining to the accelerometer frame and proof mass, respectively, as shown in Fig. 13. From this, we may reference the proof-mass point-displacement response magnitudes with the

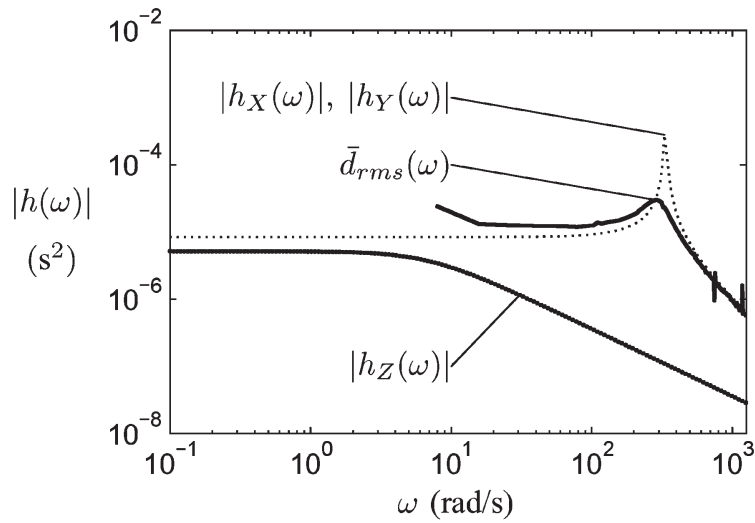

(a)

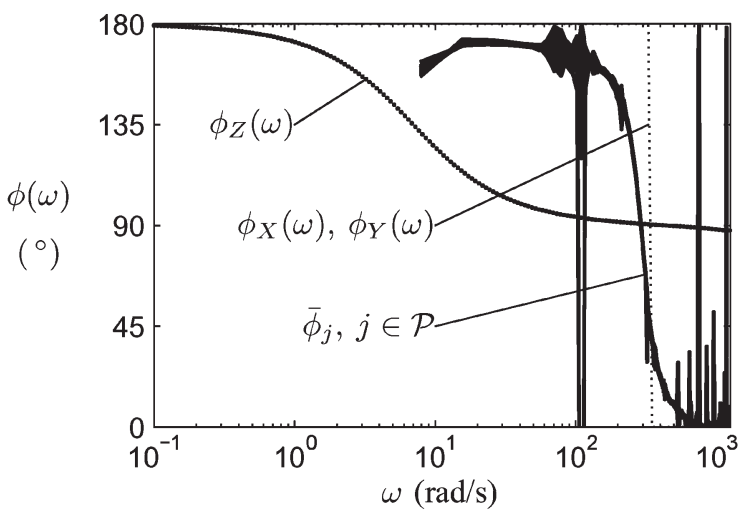

(b)

Fig. 14. Comparison between the SBA model and the experimental results. (a) Magnitudes. (b) Phase angles.

rms value of the frame point-acceleration response magnitudes. This yields the normalized displacements

$$
\bar{d}_{j}(\omega)=\frac{d_{j}(\omega)}{\sqrt{\frac{1}{n} \sum_{k \in \mathcal{F}}\left|a_{k}(\omega)\right|^{2}}} \quad j \in \mathcal{P}
$$

where $n$ is the cardinality of $\mathcal{F}$. Finally, we obtain an overall magnitude response of the proof mass by taking the rms value of the displacements of its 60 scanned points. Symbolically, we have

$$
\bar{d}_{\mathrm{rms}}(\omega)=\sqrt{\frac{1}{m} \sum_{j \in \mathcal{P}}\left|\bar{d}_{j}(\omega)\right|^{2}}
$$

where $m=60$ is the cardinality of $\mathcal{P}$. On the other hand, the meaning of an overall phase diagram of the proof-mass point displacements is less apparent, and therefore, it was decided to leave them separate. Hence, the phase angle $\bar{\phi}_{j}(\omega)$ of point $j \in \mathcal{P}$ is readily computed as

$$
\bar{\phi}_{j}=\arctan \left[\frac{\Im\left\{\bar{d}_{j}(\omega)\right\}}{\Re\left\{\bar{d}_{j}(\omega)\right\}}\right] .
$$

The resulting frequency response is shown in Fig. 14(a) and (b), along with the modeled frequency response, which was already shown in Fig. 10. Notice that the 60 phase angles $\bar{\phi}_{j}$, $j \in \mathcal{P}$ are shown in Fig. 14(b). As shown in these figures, the 
measured frequency response is fairly close to the ones given by the transfer functions $h_{X}(\omega)$ and $h_{Y}(\omega)$. The modeled and measured peak frequencies are 53.2 and $46.3 \mathrm{~Hz}$, respectively. This difference may be due in part to the rough approximation of the damping coefficient, which appears to have been underestimated.

\section{CONCLUSION}

Although the assumptions put forward in this paper are common in elastodynamics modeling, the mathematical tools used are not. In particular, screw theory allows for a sound, broad-scope, and simple formulation of the dynamic model of a lumped MEMS. The general framework in which the proposed method is developed is important for the evaluation of large numbers of variants with different topologies, numbers of degrees of freedom, etc. Moreover, the simplicity of the resulting model formulation is thought to be of crucial importance, since the usefulness of a symbolic solution is generally dictated by its level of intricacy. A major factor contributing to the simplicity of the dynamic model obtained is the parameters chosen for the representation of rigid-link rotations in space. As shown in (5), for small displacements, the time rates of these parameters are approximately equal to the rigid-link angular velocity, which simplifies the model formulation. The resulting symbolic models should yield information on the relations between the design parameters and the design objectives. This is thought to be particularly important in MEMS design, e.g., for assessing the scalability of a design, the effect of constraints on certain dimensions due to microfabrication limitations, the overall feasibility of certain actuation schemes, estimating parasitic displacements, etc.

The model was applied to the relatively simple mechanical architecture of the already-existing ADXL150 accelerometer, and the estimated dynamic properties of the device were compared to published data, which confirmed the accuracy of the proposed model. The dynamic model was then applied to the SBA, an accelerometer that comprises 4 rigid and 18 compliant links. The modeled and measured dynamic properties of the mechanism were compared, which corroborated the previous results. Furthermore, the two case studies showed that the proposed formulation can streamline both symbolic and numeric calculations when the complete system dynamics is to be modeled.

An example of a potential application of this method to MEMS synthesis comes from the expression of the stiffness matrix obtained in (28), which is completely decoupled. Indeed, $\mathbf{K}$ appears as a product of matrices $\mathbf{R}, \mathbf{A}$, and $\mathbf{B}$, containing the information on the rigid-link poses, the mechanism topology, and the compliant-link stiffness properties, respectively. This should prove useful for the synthesis of lumped-compliance micromechanisms. As an example, one could impose the rigidlink poses and the compliant links used for a particular MEMS and then treat the topology $\mathbf{A}$ as a design variable. Another potential application of this method could come from its combination with the model proposed in [28]. In this reference, the authors modeled the dynamics of a compliant micromechanism subjected to nonlinear external forces by approximating its displacements with a time-varying linear combination of its modes. These modes are computed from a linear dynamic model similar to that of (33), except that the compliant links are discretized rather than treated as continua. Treating the compliant links as Euler-Bernoulli beams-or any other compliance model-and expressing the system state in terms of rigid-link poses, twists, and twist-rates minimizes the number of generalized coordinates of the associated model. Since computing the stiffness matrices of Euler-Bernoulli beams is a linear process, and because the dimension of the associated nonlinear eigenvalue problem is minimized, this method should streamline the computation of the mechanism dynamic response.

\section{ACKNOWLEDGMENT}

The authors would like to thank the McGill Microfabrication Laboratory (funded by CFI, NSERC, VRQ, NanoQuebec, and the Quebec Ministry of Economic Development, Innovation and Export Trade) for the assistance in preparing prototypes of the SBA analyzed in Section IV.

\section{REFERENCES}

[1] H. Qu, D. Fang, and H. Xie, "A monolithic CMOS-MEMS 3-axis accelerometer with a low-noise, low-power dual chopper amplifier," in Proc. Solid-State Sens., Actuators Microsyst. Workshop. Hilton Head Island, SC, Jun. 2006, pp. 224-227.

[2] K. A. Jensen, C. P. Lusk, and L. L. Howell, "An xyz micromanipulator with three translational degrees of freedom," Robotica, vol. 24 , no. 3 , pp. 305-314, May 2006.

[3] S. D. Senturia, N. Aluru, and J. White, "Simulating the behavior of MEMS devices: Computational methods and needs," IEEE Comput. Sci. Eng., vol. 4, no. 1, pp. 30-43, Jan.-Mar. 1997.

[4] K.-J. Bathe, Finite Element Procedures in Engineering Analysis. Englewood Cliffs, NJ: Prentice-Hall, 1982.

[5] B. D. Jensen, M. P. de Boer, N. D. Masters, F. Bitsie, and D. A. La Van, "Interferometry of actuated microcantilevers to determine material properties and test structure nonidealities in MEMS," J. Microelectromech. Syst., vol. 10, no. 3, pp. 336-346, Sep. 2001.

[6] S. K. De and N. R. Aluru, "Full-Lagrangian schemes for dynamic analysis of electrostatic MEMS," J. Microelectromech. Syst., vol. 13, no. 5, pp. 737-758, Oct. 2004.

[7] E. Wittbrodt, I. Adamiec-Wójcik, and S. Wojciech, Dynamics of Flexible Multibody Systems, ser. Foundations of Engineering Mechanics. Berlin, Germany: Springer-Verlag, 2006.

[8] U. Kirsch, "Optimal topologies of structures," Appl. Mech. Rev., vol. 42, no. 8, pp. 223-239, 1989.

[9] K.-J. Lu and S. Kota, "Topology and dimensional synthesis of compliant mechanisms using discrete optimization," ASME J. Mech. Des., vol. 128, no. 5, pp. 1080-1091, Sep. 2006.

[10] S. Nishiwaki, M. I. Frecker, S. Min, and N. Kikuchi, "Topology optimization of compliant mechanisms using the homogenization method," Int. J. Numer. Methods Eng., vol. 42, no. 3, pp. 535-559, Jun. 1998.

[11] L. L. Howell, Compliant Mechanisms. New York: Wiley, 2001.

[12] S. M. Lyon, P. A. Erickson, M. S. Evans, and L. L. Howell, "Prediction of the first modal frequency of compliant mechanisms using the pseudorigid-body model," ASME J. Mech. Des., vol. 121, no. 2, pp. 309-313, Jun. 1999.

[13] Y.-Q. Yu, L. L. Howell, C. Lusk, Y. Yue, and M.-G. He, "Dynamic modeling of compliant mechanisms based on the pseudo-rigid-body model," ASME J. Mech. Des., vol. 127, no. 4, pp. 760-765, Jul. 2005.

[14] L. Meirovitch, Analytical Methods in Vibrations. New York: Macmillan, 1967.

[15] J. M. Selig and X. Ding, "A screw theory of static beams," in Proc. IEEE/RSJ Int. Conf. Intell. Robots Syst., Maui, HI, 2001, pp. 312-317.

[16] A. A. Shabana, "Flexible multibody dynamics: Review of past and recent developments," Multibody Syst. Dyn., vol. 1, no. 2, pp. 189-222, Jun. 1997. 
[17] P. Stellman, T. Buchner, W. J. Arora, and G. Barbastathis, "Dynamics of nanostructured origami," J. Microelectromech. Syst., vol. 16, no. 4, pp. 932-949, Aug. 2007.

[18] S. Senturia, Microsystem Design. Boston, MA: Kluwer, 2001.

[19] J. Angeles, Fundamentals of Robotic Mechanical Systems, 3rd ed. New York: Springer-Verlag, 2007.

[20] R. J. Roark and W. C. Young, Formulas for Stress and Strain, 5th ed. New York: McGraw-Hill, 1975.

[21] J. M. Selig, Geometrical Methods in Robotics, ser. Monographs in Computer Science. New York: Springer-Verlag, 1996.

[22] J. Angeles, K. E. Zanganeh, and S. Ostrovskaya, "The analysis of arbitrarily-damped linear mechanical systems," Arch. Appl. Mech., vol. 69, no. 8, pp. 529-541, 1999.

[23] B. R. Munson, D. F. Young, and T. H. Okiishi, Fundamentals of Fluid Mechanics, 5th ed. Hoboken, NJ: Wiley, 2006.

[24] P. Cardou and J. Angeles, "Simplectic architectures for true multi-axial accelerometers: A novel application of parallel robots," in Proc. IEEE Int. Conf. Robot. Autom., Rome, Italy, Apr. 2007, pp. 181-186.

[25] M. L. Culpepper and G. Anderson, "Design of a low-cost nanomanipulator which utilizes a monolithic, spatial compliant mechanism," Precis. Eng., vol. 28, no. 4, pp. 469-482, Oct. 2004.

[26] J. J. Wortman and R. A. Evans, "Young's modulus, shear modulus, and Poisson's ratio in silicon and germanium," J. Appl. Phys., vol. 36, no. 1, pp. 153-156, Jan. 1965.

[27] W. D. Pilkey, Formulas for Stress, Strain, and Structural Matrices, 2nd ed. Hoboken, NJ: Wiley, 2005.

[28] G. K. Ananthasuresh, R. K. Gupta, and S. D. Senturia, "An approach to macromodeling of MEMS for nonlinear dynamic simulation," in Proc. ASME-MEMS, 1996, vol. 59, pp. 401-407.

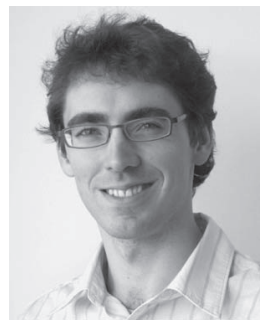

Philippe Cardou (S'07-M'07) received the degree in mechanical engineering from Laval University, Quebec City, PQ, Canada, in 2003, and the Ph.D. degree in mechanical engineering from McGill University, Montreal, PQ, in 2008.

Since September 2007, he has been an Assistant Professor with the Department of Mechanical Engineering, Laval University, where he is a member of the Robotics Laboratory. His main interests are related to robotics: kinematics in general, and in particular, the estimation of the rigid-body acceleration field from accelerometer measurements; compliant mechanism design; and cable-driven mechanism design.

Dr. Cardou is a member of the American Society of Mechanical Engineers.

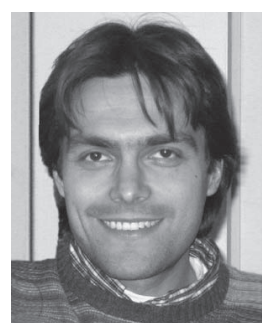

Damiano Pasini (M'06) received M.S. degrees in civil engineering and in architectural engineering, from the University of Pavia, Pavia, Italy, in 1995 and 2000, respectively, and the Ph.D. degree in mechanical engineering, with a thesis on the optimization of material, shape, and structural form, from the University of Bristol, Bristol, U.K., in 2003.

From 1995 to 2000, he was a Professional Civil Engineer in Italy. In January 2003, he joined the Department of Adaptronics, Institute of Structural Mechanics, German Aerospace Center, Braunschweig, Germany, where his research focused on the optimization of solid-state hinges for adaptive wing flaps. Since July 2004, he has been an Assistant Professor with the Department of Mechanical Engineering, McGill University, Montreal, PQ, Canada. His research is in the area of optimum design, multiscale modeling, and biomimetics.

Dr. Pasini is a member of the McGill Institute for Advanced Materials and the American Society of Mechanical Engineers.

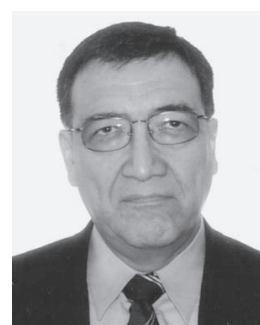

Jorge Angeles (SM'90-F'06) received the Dipl.Eng. degree in electromechanical engineering and the M.Eng. degree in mechanical engineering from the Universidad Nacional Autonoma de Mexico, Mexico City, Mexico, in 1969 and 1970, respectively, and the Ph.D. degree in applied mechanics from Stanford University, Stanford, CA, in 1973.

In 1984, he joined the Department of Mechanical Engineering, McGill University, Montreal, PQ, Canada, where in 1985, he became a founding member of the Center for Intelligent Machines and is currently James McGill Professor of Mechanical Engineering, and the Founder and Director of the Robotic Mechanical Systems Laboratory. He is the author or coauthor of various books on kinematics and dynamics of mechanical systems plus numerous technical papers published in research journals and conference proceedings. He works on the theoretical and computational aspects of robotic mechanical systems, in addition to design theory and methodology. He is the holder of three international patents in the area of mechanical systems.

Dr. Angeles is a Fellow the of the RSC, The Academies of Arts, Humanities and Sciences of Canada. He is one of the 12 honorary members of IFToMM the International Federation for the Promotion of Mechanism and Machine Science. He is a Fellow of the Canadian Society for Mechanical Engineers and the American Society of Mechanical Engineers. His professional registration as an Engineer includes Quebec, Mexico, and Germany. 